TRANSACTIONS OF THE

AMERICAN MATHEMATICAL SOCIETY

Volume 351, Number 5, Pages 1895-1918

S 0002-9947(99)02304-1

Article electronically published on January 27, 1999

\title{
LINKING FORMS, RECIPROCITY FOR GAUSS SUMS AND INVARIANTS OF 3-MANIFOLDS
}

\author{
FLORIAN DELOUP
}

\begin{abstract}
We study invariants of 3-manifolds derived from finite abelian groups equipped with quadratic forms. These invariants arise in Turaev's theory of modular categories and generalize those of H. Murakami, T. Ohtsuki and M. Okada. The crucial algebraic tool is a new reciprocity formula for Gauss sums, generalizing classical formulas of Cauchy, Kronecker, Krazer and Siegel. We use this reciprocity formula to give an explicit formula for the invariants and to generalize them to higher dimensions.
\end{abstract}

\section{INTRODUCTION}

1.0. Overview. Let $M$ be a closed oriented 3-manifold. We consider a $\mathbf{C}$-valued topological invariant $\tau(M ; G, q)$ depending on a finite abelian group $G$ equipped with a quadratic form $q: G \rightarrow \mathbf{Q} / \mathbf{Z}$. This invariant arises in the theory of modular categories (see [Tu1, Chap. 1]) and generalizes an invariant introduced by $\mathrm{H}$. Murakami, T. Ohtsuki and M. Okada [MOO].

The aim of the paper is to compute $\tau(M ; G, q)$ in terms of classical invariants and to describe its main properties. In particular, $\tau(M ; G, q)$ is completely determined by $(G, q)$, the first Betti number of $M$ and the linking form of $M$ (Theorems 1 and 4 ). We also compute the absolute value of $\tau(M ; G, q)$ (Theorem 1) which only depends on the order of a certain cohomology group of $M$.

The crucial algebraic result of this paper is a new reciprocity formula for Gauss sums (Theorem 3). It allows us to establish an explicit formula for the invariant $\tau(M ; G, q)$ (Theorem 4). As another application of the reciprocity formula, we generalize the invariant $\tau(M ; G, q)$ to closed oriented $(4 n-1)$-manifolds. Here we apply the reciprocity formula in a topological context but we expect it to have algebraic applications as well.

1.1. Definition of $\tau(M ; G, q)$ and first properties. Fix a finite abelian group $G$. A quadratic form $q: G \rightarrow \mathbf{Q} / \mathbf{Z}$ is a function satisfying $q(n x)=n^{2} q(x)$ for any $n \in \mathbf{Z}$ and $x \in G$ and such that the function defined by $b_{q}(x, y)=q(x+y)-q(x)-q(y)$ is a (symmetric) bilinear form on $G$, called the bilinear form associated to $q$. Let $\operatorname{ad} b_{q}: G \rightarrow \operatorname{Hom}(G, \mathbf{Q} / \mathbf{Z})$ denote the adjoint homomorphism of $b_{q}$. We define the

Received by the editors April 22, 1997.

1991 Mathematics Subject Classification. Primary 11E81, 57N10.

Key words and phrases. Reciprocity, quadratic form, Gauss sum, Witt group, manifold, linking form, modular category. 
Gauss sum by

$$
\gamma(G, q)=\left|\operatorname{ker} a d b_{q}\right|^{-1 / 2}|G|^{-1 / 2} \sum_{x \in G} e^{2 \pi i q(x)} .
$$

Here the normalization factor $\left|\operatorname{ker} \operatorname{ad} b_{q}\right|^{-1 / 2}|G|^{-1 / 2}$ ensures that $\gamma(G, q)$ is either 0 or an 8-th root of unity ([Sc, chapter 5]). The following lemma gives a necessary and sufficient condition for $\gamma(G, q)$ to vanish (the proof is given in $\S 2.3$ ).

\section{Lemma 1.1.}

$$
|\gamma(G, q)|= \begin{cases}0 & \text { if } q\left(\operatorname{ker} \operatorname{ad} b_{q}\right) \neq 0 \\ 1 & \text { if } q\left(\operatorname{ker} \operatorname{ad} b_{q}\right)=0\end{cases}
$$

It is not hard to see that if $q$ is non-degenerate or if $|G|$ is odd, then $q\left(\operatorname{ker} \operatorname{ad} b_{q}\right)=$ 0 and hence $\gamma(G, q) \neq 0$.

Let $M$ be a closed connected oriented 3-manifold. There is a simply connected compact smooth 4-manifold $W$ such that $\partial W=M$ (see [Rok]). As a consequence of Poincaré duality, the second homology group of $W$ is a free abelian group and carries a symmetric bilinear pairing $B_{W}: H_{2}(W ; \mathbf{Z}) \times H_{2}(W ; \mathbf{Z}) \rightarrow \mathbf{Z}$ (which may be degenerate, since $W$ has a boundary). Let $\sigma\left(B_{W}\right)$ be the signature of $B_{W}$, which is equal to the number of positive eigenvalues of $B_{W}$ minus the number of negative eigenvalues of $B_{W}$. Denote by $b_{2}(W)$ the second Betti number of $W$.

For any pair $(G, q)$ such that $\gamma(G, q) \neq 0$, we define the following complex number:

$$
\tau(M ; G, q)=\overline{\gamma(G, q)}^{\sigma\left(B_{W}\right)}\left(|G|\left|\operatorname{ker} \operatorname{ad} b_{q}\right|\right)^{-\frac{b_{2}(W)}{2}} \sum_{x \in G \otimes H_{2}(W ; \mathbf{Z})} e^{2 \pi i\left(q \otimes B_{W}\right)(x)} .
$$

Bar denotes complex conjugate. Here $q \otimes B_{W}$ denotes the $\mathbf{Q} / \mathbf{Z}$-valued quadratic form on $G \otimes H_{2}(W ; \mathbf{Z})$ defined by $\left(q \otimes B_{W}\right)\left(\sum_{j} x_{j} \otimes y_{j}\right)=\sum_{j} q\left(x_{j}\right) B_{W}\left(y_{j}, y_{j}\right)+$ $\sum_{j<k} b_{q}\left(x_{j}, x_{k}\right) B_{W}\left(y_{j}, y_{k}\right)$ where $x_{j} \in G, y_{j} \in H_{2}(W ; \mathbf{Z})$. See [MH] for further details. The terms $\overline{\gamma(G, q)}^{\sigma\left(B_{W}\right)}$ and $\left(|G|\left|\operatorname{ker} \operatorname{ad} b_{q}\right|\right)^{-\frac{b_{2}(W)}{2}}$ in the right hand side of (2) are normalization factors which are better understood in light of Theorem 1 below. Theorem 1 says that the complex number we have defined does not depend on the choice of $W$, which in particular justifies the fact that we made the notation dependent on $M$ rather than on $W$ in formula (2).

Before we state Theorem 1, we recall a few facts about the linking form $\mathcal{L}_{M}$ of an oriented and closed 3-manifold $M$. Let $u, v$ be two transversal singular 1-cycles in $M$ respectively representing torsion elements $[u],[v]$ in $H_{1}(M ; \mathbf{Z})$. There exists $n \in \mathbf{Z}$ such that $n v=\partial C$, where $C$ is a singular 2-chain in $M$. Then the linking form $\mathcal{L}_{M}$ is the bilinear pairing defined on Tors $H_{1}(M ; \mathbf{Z})$ by

$$
\mathcal{L}_{M}([u],[v])=\frac{u \cdot C}{n} \bmod 1
$$

where $u \cdot C$ denotes the intersection number of $u$ and $C$ in $M$. The fact that $[u]$ is a torsion element ensures that the right hand side does not depend on the particular choice of $C$. Since a change of representatives for $[u]$ and $[v]$ will change $u \cdot C$ only up to a multiple of $n$, the right hand side only depends on $[u]$ and $[v]$. Properties of intersection numbers in odd dimension ensure that $\mathcal{L}_{M}$ is symmetric. It is a consequence of Poincaré duality that $\mathcal{L}_{M}$ is non-degenerate. 
Theorem 1. The complex number $\tau(M ; G, q)$ is a topological invariant of $M$, independent of the choice of $W$. If the pair $(G, q)$ is fixed, $\tau$ is completely determined by the following data:

(i) the first Betti number, $\operatorname{dim} H_{1}(M ; \mathbf{R})$;

(ii) the linking form $\mathcal{L}_{M}$ on Tors $H_{1}(M ; \mathbf{Z})$, considered up to isomorphism.

Moreover, if $\tau(M ; G, q) \neq 0$, then $\frac{\tau(M ; G, q)}{|\tau(M ; G, q)|}$ is an 8-th root of unity and the phase of $\tau(M ; G, q)$ only depends on the linking form $\mathcal{L}_{M}$ on Tors $H_{1}(M ; \mathbf{Z})$.

A useful expression for $\tau(M ; G, q)$ can be obtained by choosing $W$ as follows. Present the 3-manifold $M$ as the result of surgery in $S^{3}=\partial B^{4}$ on a framed link $L$ with components $L_{1}, \ldots, L_{m}$. Let $W$ be the simply connected compact smooth 4-manifold obtained by attaching $m$ 2-handles to the 4-ball $B^{4}$ (the attaching map being determined by the framed link $L$ ). These $m 2$-handles yield a basis of $H_{2}(W ; \mathbf{Z})$ (which is free of rank $m$ ). The intersection form $B_{W}$, with respect to this basis, is given by an $(m \times m)$ matrix of integers (whose $(j, k)$-entry is the linking number of $L_{j}$ and $\left.L_{k}\right)$. The definition $(2)$ of $\tau(M ; G, q)$ can be rewritten in terms of the linking matrix $A=\left(l_{j k}\right)_{1 \leq j, k \leq m}$ for $L$ :

$$
\tau(M ; G, q)=\overline{\gamma(G, q)}^{\sigma(A)}\left(|G|\left|\operatorname{ker} \operatorname{ad} b_{q}\right|\right)^{-\frac{m}{2}} \sum_{x \in G \otimes \mathbf{Z}^{m}} e^{2 \pi i(q \otimes A)(x)},
$$

where $q \otimes A: G \otimes \mathbf{Z}^{m} \rightarrow \mathbf{Q} / \mathbf{Z}$ denotes the tensor product of the quadratic form $q$ and the symmetric bilinear form defined by $A$. See $[\mathrm{MH}]$ for details.

The invariants $M \longmapsto \tau(M ; G, q)$ arise in the theory of modular categories (see [Tu1]). We refer to the appendix $A$ for the construction of $\tau(M ; G, q)$ from a modular category.

These invariants also generalize the invariants $M \mapsto Z_{N}(M ; \omega)$ introduced by $\mathrm{H}$. Murakami, T. Ohtsuki and M. Okada [MOO] and further studied by J. Mattes, M. Polyak and N. Reshetikhin (see $[\mathrm{MPR}]$ ). Here $N$ is a positive integer and $\omega$ is an $N$-th primitive root of unity (resp. $2 N$-th primitive root of unity) if $N$ is odd (resp. if $N$ is even). The relation is as follows: $Z_{N}(M, \omega)=\tau(M ; G, q)$ where $G=\mathbf{Z} / N \mathbf{Z}$ and the quadratic form $q: G \rightarrow \mathbf{Q} / \mathbf{Z}$ is chosen so that $\omega=\exp (2 \pi i q(1 \bmod N))$.

One property of $\tau$ is the multiplicativity on connected sums. Let $M \# M^{\prime}$ denote the connected sum of two closed oriented 3-manifolds $M$ and $M^{\prime}$. Then

$$
\tau\left(M \# M^{\prime} ; G, q\right)=\tau(M ; G, q) \cdot \tau\left(M^{\prime} ; G, q\right) .
$$

Another property is the behavior of $\tau$ under a reversal of orientation. Let $M$ be a closed oriented 3-manifold and let $-M$ denote the same manifold with the orientation reversed. Then

$$
\tau(-M ; G, q)=\overline{\tau(M ; G, q)} .
$$

Note also that $\tau$ is multiplicative with respect to orthogonal sums of pairs $(G, q)$ of finite abelian groups equipped with quadratic forms. All these properties follow from the definition of $\tau$ and elementary properties of Gauss sums.

Elementary considerations show that we can always assume, without loss of generality, that $q$ is non-degenerate. More precisely we have

Lemma 1.2. Let $G$ be a finite abelian group equipped with a quadratic form $q$ such that $\gamma(G, q) \neq 0$. Then

$$
\tau(M ; G, q)=\tau\left(M ; G / \operatorname{ker} \operatorname{ad} b_{q}, \tilde{q}\right)
$$


where $\tilde{q}$ is the non-degenerate quadratic form on $G / \operatorname{ker} \operatorname{ad} b_{q}$ induced by $q$.

The following theorem computes the absolute value of $\tau$.

Theorem 2. Let $M$ be a closed oriented 3-manifold. If $\tau(M ; G, q) \neq 0$, then

$$
|\tau(M ; G, q)|=\left|H^{1}\left(M ; G / \operatorname{ker} \operatorname{ad} b_{q}\right)\right|^{1 / 2} .
$$

In particular, the absolute value of $\tau(M ; G, q)$ does not depend on the quadratic form $q$ unless $q$ is degenerate.

Using Theorem 2, one can rewrite $\tau(M ; G, q)$ as a product of Gauss sums normalized as in (1):

$$
\tau(M ; G, q)=\overline{\gamma(G, q)}^{\sigma\left(B_{W}\right)} \gamma\left(G \otimes H_{2}(W ; \mathbf{Z}), q \otimes B_{W}\right)\left|H^{1}(M ; \tilde{G})\right|^{\frac{1}{2}},
$$

where $\tilde{G}=G / \operatorname{ker}$ ad $b_{q}$.

Necessary and sufficient conditions for $\tau(M ; G, q)$ to vanish are given in Theorem 6 (see $\S 3.4$ ). Theorems 1 and 2 indicate that the interesting topological information is concentrated in the phase of $\tau(M ; G, q)$. The question arises as to how to determine its algebraic dependence on $q$ and $\mathcal{L}_{M}$. Theorem 1 shows that if $\tau(M ; G, q)$ is not zero, the phase can take at most 8 values. In fact, we can show that

$$
\frac{\tau(M ; G, q)}{\left|H^{1}(M ; \tilde{G})\right|^{\frac{1}{2}}}
$$

depends on $q$ only modulo hyperbolic quadratic forms and on $\mathcal{L}_{M}$ only modulo hyperbolic symmetric bilinear forms. See Theorem $5, \S 3.3$ for a precise statement.

1.2. The reciprocity formula. Further study of the invariant $\tau(M ; G, q)$ is based on a new reciprocity formula for Gauss sums. The following reciprocity formula goes back to the 19-th century:

Lemma 1.3 (Cauchy, Kronecker). Let $a$ and $b$ be two nonzero integers.

$$
|b|^{-\frac{1}{2}} \sum_{x \in \mathbf{Z} / b \mathbf{Z}} e^{\pi i \frac{a}{b} x^{2}+\pi i a x}=e^{\frac{\pi i}{4}(\operatorname{sign}(a b)-a b)}|a|^{-\frac{1}{2}} \sum_{x \in \mathbf{Z} / a \mathbf{Z}} e^{-\pi i \frac{b}{a} x^{2}+\pi i b x} .
$$

An analytical proof of this lemma can be found in [Ch, Chapter IX], where some historical background is given. Early proofs, due to Cauchy and Kronecker, are analytical; one of them consists in studying the limiting case of a transformation formula for the theta-function $\theta_{3}(u, \tau)=\sum_{n=-\infty}^{n=\infty} e^{\pi i n^{2} \tau+2 \pi i u}$. Another reciprocity formula appears as an important step of $\mathrm{H}$. Braun's classification of quadratic forms in [Br]. We formulate it as follows. Let $A$ be a symmetric $m \times m$ matrix of integers and let $r$ (resp. $\sigma(A)$ ) be the rank (resp. the signature) of $A$. There exists a matrix $A^{\prime}$ with integer entries and nonzero determinant and a unimodular matrix $P$ such that $P^{t} A P=A^{\prime} \oplus\left(0_{m-r}\right)$ where $0_{m-r}$ is the zero matrix of size $m-r[\mathrm{Ky}$, lemma $1]$.

Lemma 1.4. Let $d$ be a nonzero integer. Assume that either $d$ is even or $A$ is even (i.e., its diagonal entries are even). Then

$$
d^{-\frac{m}{2}} \sum_{x \in(\mathbf{Z} / d \mathbf{Z})^{m}} e^{\frac{\pi i x^{t} A x}{d}}=\frac{d^{\frac{m-r}{2}} e^{\frac{\pi i}{4} \sigma(A)}}{\left|\operatorname{det} A^{\prime}\right|^{\frac{1}{2}}} \sum_{y \in \mathbf{Z}^{m} / A^{\prime} \mathbf{Z}^{m}} e^{-\pi i d y^{t} A^{\prime-1} y} .
$$


According to $[\mathrm{Br}]$, the formula (9) is due to A. Krazer $[\mathrm{Kr}]$. The proof is analytical and also involves the limiting case of a transformation formula for thetafunctions. A particular case of (9) also appears in the work of C. Siegel [Si] in the context of modular transformations. The formula (9) is discussed in [MPR, lemma (8.5)], with a slight imprecision. Recently, R. Dabrowski [Dab] found a proof of (9) using $p$-adic numbers, in which analysis is kept to a minimum. Note that (8) is not a particular case of (9).

In order to generalize both formulas (8) and (9) to our setting, we need a construction relating symmetric bilinear forms on free abelian groups to bilinear and quadratic forms on finite abelian groups. This is a particular case of a correspondence between isomorphism classes of bilinear (resp. quadratic) forms on modules over a Dedekind ring $R$ and isomorphism classes of bilinear (resp. quadratic) forms with values in $\bar{R} / R$ where $\bar{R}$ denotes the quotient field of $R$. This correspondence was studied by C.T.C. Wall, M. Kneser, A. Durfee and others. We refer to [Du, §2] for the general construction and further references.

A lattice is defined as a finitely generated free abelian group. A symmetric bilinear form $f$ on a lattice $F$ gives rise to a symmetric bilinear form $L_{f}$ on Tors(coker ad $f$ ), where ad $f$ is the homomorphism $F \rightarrow \operatorname{Hom}(F, \mathbf{Z})$ adjoint to $f$. The construction is as follows. The homomorphism ad $f$ induces a homomorphism $a_{f}: F \otimes \mathbf{Q} \rightarrow \operatorname{Hom}(F, \mathbf{Q})$. Set $K=\operatorname{Hom}(F, \mathbf{Z}) \cap \operatorname{Im} a_{f}$. Set

$$
K_{f}=K / \operatorname{Im} \text { ad } f=\operatorname{Tors}(\text { coker } \operatorname{ad} f) .
$$

The formula

$$
L_{f}(x+\operatorname{Im} \operatorname{ad} f, y+\operatorname{Im} \operatorname{ad} f)=x_{\mathbf{Q}}(\tilde{y}) \bmod \mathbf{Z}
$$

where $x, y \in K, x_{\mathbf{Q}}$ denotes the rational extension of $x$ and $\tilde{y} \in a_{f}^{-1}(y)$, does not depend on the choice of the lift $\tilde{y}$ and defines a non-degenerate symmetric form $L_{f}: K_{f} \times K_{f} \rightarrow \mathbf{Q} / \mathbf{Z}$.

The form $f: F \times F \rightarrow \mathbf{Z}$ on $F$ is said to be even if $f(x, x) \in 2 \mathbf{Z}$ for all $x \in F$, odd otherwise. A quadratic form $Q$ is said to be over a symmetric bilinear form $B$ if $B$ is the bilinear form associated to $Q$. In the case when $f$ is even, one can unambiguously define a quadratic form $\phi_{f}: K_{f} \rightarrow \mathbf{Q} / \mathbf{Z}$ over $L_{f}$ by the formula

$$
\phi_{f}(x+\operatorname{Im} \operatorname{ad} f)=\frac{1}{2} x_{\mathbf{Q}}(\tilde{x}) \bmod \mathbf{Z}
$$

where $x \in K, x_{\mathbf{Q}}$ is the rational extention of $x$ and $\tilde{x} \in a_{f}^{-1}(x)$.

The construction of $\phi_{f}$ can be generalized in terms of Wu classes. A Wu class for $f$ is an element $w \in F$ such that $f(w, x) \equiv f(x, x) \bmod 2$, for any $x \in F$. In particular, $f$ is even if and only if 0 is a Wu class for $f$. Given a Wu class $w$ for $f$, one can associate a quadratic form $\phi_{f, w}: K_{f} \rightarrow \mathbf{Q} / \mathbf{Z}$ over $L_{f}$ by

$$
\phi_{f, w}(x+\operatorname{Im} \operatorname{ad} f)=\frac{1}{2}\left(x_{\mathbf{Q}}(\tilde{x})-x(w)\right) \bmod \mathbf{Z} .
$$

Clearly, $\phi_{f, 0}=\phi_{f}$ if $f$ is even and $\phi_{f, w}$ depends only on $w \bmod 2$. All quadratic forms over $L_{f}$ arise as $\phi_{f, w}$ : more precisely, there is a one-to-one correspondence between quadratic forms over $L_{f}$ and Wu classes $w \in F$ modulo 2 [BM, Theorem $2.4]$.

$$
\begin{aligned}
& \text { Clearly }\left(K_{-f}, L_{-f}\right)=\left(K_{f},-L_{f}\right) \text { and } \\
& \qquad \begin{array}{l}
\left(K_{-f}, \phi_{-f, w}\right)=\left(K_{-f}, \phi_{-f,-w}\right)=\left(K_{f},-\phi_{f, w}\right) .
\end{array}
\end{aligned}
$$


It is also clear that the correspondences $f \mapsto L_{f}, f \mapsto \phi_{f}$ and $(f, w) \mapsto \phi_{f, w}$ take unimodular forms to the trivial form and preserve direct sums. In general, they do not preserve the tensor product.

Let $f: V \times V \rightarrow \mathbf{Z}$ and $g: W \times W \rightarrow \mathbf{Z}$ be symmetric bilinear forms on lattices $V$ and $W$ respectively, equipped with Wu classes $v \in V$ and $w \in W$ respectively. We are now ready to state our reciprocity formula for the Gauss sum

$$
\gamma\left(K_{f} \otimes V, \phi_{f, v} \otimes g\right)=\left|\operatorname{ker} \operatorname{ad}\left(L_{f} \otimes g\right)\right|^{-\frac{1}{2}}\left|K_{f} \otimes W\right|^{-\frac{1}{2}} \sum_{x \in K_{f} \otimes W} e^{2 \pi i\left(\phi_{f, v} \otimes g\right)(x)} .
$$

We recall that $\phi_{f, v} \otimes g$ denotes the quadratic form $K_{f} \otimes W \rightarrow \mathbf{Q} / \mathbf{Z}$ uniquely determined by $\left(\phi_{f, v} \otimes g\right)(x \otimes y)=\phi_{f, v}(x) g(y, y), x \in K_{f}, y \in W$.

Theorem 3 (Reciprocity formula). The following relation holds:

$$
\gamma\left(K_{f} \otimes W, \phi_{f, v} \otimes g\right)=e^{\frac{\pi i}{4}(\sigma(f) \sigma(g)-f(v, v) g(w, w))} \overline{\gamma\left(K_{g} \otimes V, \phi_{g, w} \otimes f\right)} .
$$

Note the symmetry in $f$ and $g$ in (13).

In the case when one of the $\mathrm{Wu}$ classes is 0 (which implies that one of the forms is even), the formula (13) simplifies. We denote by $q_{L_{f}}$ the quadratic form defined by $q_{L_{f}}(x)=L_{f}(x, x) ; \frac{1}{2} g$ denotes the symmetric bilinear form $W \times W \rightarrow \frac{1}{2} \mathbf{Z},(x, y) \mapsto$ $\frac{1}{2} g(x, y)$.

Corollary. Suppose that $g$ is even. Then

$$
\gamma\left(K_{f} \otimes W, q_{L_{f}} \otimes \frac{1}{2} g\right)=e^{\frac{\pi i}{4} \sigma(f) \sigma(g)} \overline{\gamma\left(K_{g} \otimes V, \phi_{g} \otimes f\right)} .
$$

Proof. We have $\phi_{g, 0}=\phi_{g}$. Let now $Q$ be any quadratic form over $L_{f}$. Since $L_{f}(x, x)=2 Q(x)$, we have $q_{L_{f}} \otimes \frac{1}{2} g=Q \otimes g$. Choose $Q=\phi_{f, v}$ and apply (13).

Formula (13) generalizes the formulas (8) and (9). Formula (8) is the particular case of (13) when both $f$ and $g$ are 1-dimensional and is used in the proof of Theorem 3. The reciprocity formula gives a new proof of (9), which can be deduced from (14) as follows. In the case when $A$ is even in (9), set $g=A$, choose $f: \mathbf{Z} \times \mathbf{Z} \rightarrow \mathbf{Z},(x, y) \mapsto d x y$ and apply (14). The case when $d$ is even in (9) is treated similarly by exchanging the roles of $f$ and $g$ in formula (14).

1.3. The main theorem. This section is devoted to the application of the reciprocity formula (13) to the study of the invariant $\tau(M ; G, q)$. Let us denote by $T$ the finite abelian group Tors $H_{1}(M ; \mathbf{Z})$. Recall that $\mathcal{L}_{M}$ denotes the linking form on $T$.

Theorem 4. Let $f: F \times F \rightarrow \mathbf{Z}$ be a symmetric bilinear form on a lattice $F$, with a Wu class $v \in F$ such that $\left(K_{f}, \phi_{f, v}\right)=(G, q)$. Let $Q: T \rightarrow \mathbf{Q} / \mathbf{Z}$ be a quadratic form over $\mathcal{L}_{M}$. Then

$$
\tau(M ; G, q)=\overline{\gamma(T, Q)}^{f(v, v)} \gamma(T \otimes F, Q \otimes f)\left|H^{1}(M ; G)\right|^{\frac{1}{2}} .
$$

For the definition of $\phi_{f, v}$, see the previous section, $\S 1.2$.

Remarks. 1. Theorem 1 implies that the right hand side of (15) does not depend on the particular choice of $Q$.

2. Since the linking form $\mathcal{L}_{M}$ is non-degenerate, so is $Q$. By lemma $1.1, \gamma(T, Q) \neq$ 0 . 
3. It is known that there always exists a form $f: F \times F \rightarrow \mathbf{Z}$ satisfying the hypothesis of Theorem 4 (see [Du, Corollary 4.2] or lemma 2.1, part (b), §2.2).

The case when $f$ is even, with Wu class equal to 0 in (15), is interesting enough to be formulated explicitly. By $\frac{1}{2} f$, we denote the bilinear form $F \times F \rightarrow \frac{1}{2} \mathbf{Z},(x, y) \mapsto$ $\frac{1}{2} f(x, y)$; the quadratic form $q_{\mathcal{L}_{M}}$ is defined by $q_{\mathcal{L}_{M}}(x)=\mathcal{L}_{M}(x, x), x \in T$.

Corollary. For any even integral symmetric form $f: F \times F \rightarrow \mathbf{Z}$ on a lattice $F$ such that $\phi_{f}=q$, the following formula holds:

$$
\tau(M ; G, q)=\gamma\left(T \otimes F, q_{\mathcal{L}_{M}} \otimes \frac{1}{2} f\right)\left|H^{1}(M ; G)\right|^{1 / 2} .
$$

Remark. This result provides an explicit formula for $\tau(M ; G, q)$ in terms of the classical invariants of 3-manifolds listed in Theorem 1 , so that the invariant $\tau(M ; G, q)$ can be interpreted in a purely 3 -dimensional setting (compare with (2)).

Proof of Theorem 4. Using formula (7), we have

$$
\tau(M ; G, q)=\overline{\gamma(G, q)}^{\sigma\left(B_{W}\right)} \gamma\left(G \otimes H_{2}, q \otimes B_{W}\right)\left|H^{1}(M ; G)\right|^{\frac{1}{2}},
$$

where $H_{2}=H_{2}(W ; \mathbf{Z})$. Equip $B$ with a Wu class $w$ such that $Q=-\phi_{B_{W}, w}$. Then

$$
\begin{aligned}
\gamma\left(G \otimes H_{2}, q \otimes B\right) & =\gamma\left(K_{f} \otimes H_{2}, \phi_{f, v} \otimes B_{W}\right) \\
& =e^{\frac{\pi i}{4}\left(\sigma(f) \sigma\left(B_{W}\right)-f(v, v) B_{W}(w, w)\right)} \overline{\gamma\left(K_{B_{W}} \otimes F, \phi_{B, w} \otimes f\right)} \\
& =e^{\frac{\pi i}{4}\left(\sigma(f) \sigma\left(B_{W}\right)-f(v, v) B_{W}(w, w)\right)} \gamma(T \otimes F, Q \otimes f)
\end{aligned}
$$

where the first equality follows from the equality $(G, q)=\left(K_{f}, \phi_{f, v}\right)$, the second one from (13) and the last one from the fact that $\left(K_{B_{W}}, \phi_{B, w}\right)=(T,-Q)$. We now use Van der Blij's formula [Bl], which states that

$$
\gamma(G, q)=e^{\frac{\pi i}{4}(\sigma(f)-f(v, v))} .
$$

Thus

$$
\begin{aligned}
\frac{\tau(M ; G, q)}{\left|H^{1}(M ; G)\right|^{\frac{1}{2}}} & =e^{\frac{\pi i}{4} f(v, v)\left(\sigma\left(B_{W}\right)-B_{W}(w, w)\right)} \gamma(T \otimes F, Q \otimes f) \\
& =\gamma\left(T, \phi_{B, w}\right)^{f(v, v)} \gamma(T \otimes F, Q \otimes f) \\
& =\frac{{ }^{f(T, Q)}{ }^{f(v)} \gamma(T \otimes F, Q \otimes f),}{}
\end{aligned}
$$

where we used Van der Blij's formula in the first and third equalities. This is the desired result.

1.4. Plan of the paper. $\S 2$ is devoted to generalities on quadratic forms and elementary properties of Gauss sums. In $\S 3$, we prove the algebraic and topological properties of $\tau(M ; G, q)$ : Theorems 1 and 2 , the dependence of $\tau(M ; G, q)$ on $q$ and $\mathcal{L}_{M}$ modulo hyperbolic forms (Theorem 5 ) and a necessary and sufficient condition for $\tau(M ; G, q)$ to vanish (Theorem 6). The technical tool is lemma 2.1. $\S 4$ is devoted to the proof of the reciprocity formula (Theorem 3). Appendix $A$ contains an introduction to the theory of modular categories and establishes how our invariant $\tau(M ; G, q)$ can be recovered from such a category. In appendix $B$, we indicate how to define $\tau(M ; G, q)$ for a closed oriented $(4 n-1)$-manifold. 


\section{QUADRATIC FORMS ON ABELIAN GROUPS}

\subsection{The monoids $\widehat{\mathfrak{M Q}}$ and $\widehat{\mathfrak{M}}$.}

Note. This section is a very brief review of quadratic forms intended to fix notation.

Let $A$ be a lattice (resp. a finite abelian group) and let $R=\mathbf{Z}$ (resp. $\mathbf{Q} / \mathbf{Z}$ ). A quadratic form $q: A \rightarrow R$ is a function satisfying $q(n x)=n^{2} q(x)$ for any $n \in \mathbf{Z}$ and $x \in A$ and such that the function defined by $b_{q}(x, y)=q(x+y)-q(x)-q(y)$ is a bilinear form on $A$ (called the bilinear form associated to $q$ ). We say that $q$ is $n o n$ degenerate (resp. non-singular) if its associated bilinear form $b_{q}$ is non-degenerate (resp. non-singular) ${ }^{1}$. If $A$ is a finite abelian group, then $q$ is non-singular if and only if $q$ is non-degenerate.

A subgroup $N$ of $A$ is said to be orthogonal to a subgroup $N^{\prime}$ of $A$ with respect to a symmetric bilinear form $b$ if $b\left(N, N^{\prime}\right)=0$. Orthogonality for a quadratic form is defined with respect to the associated bilinear form. We say that $A$ is the orthogonal sum with respect to $b$ of two subgroups $N$ and $N^{\prime}$ if $M$ is the direct sum of $N$ and $N^{\prime}$ and $b\left(N, N^{\prime}\right)=0$. In this case, $N$ and $N^{\prime}$ are called orthogonal summands of $A$. We write $(A, b)=\left(N,\left.b\right|_{N \times N}\right) \oplus\left(N^{\prime},\left.b\right|_{N^{\prime} \times N^{\prime}}\right)$. There is a similar notation for quadratic forms. We say that a (quadratic or symmetric bilinear) form on $A$ is irreducible if $A$ has no nontrivial orthogonal summands. The negative $-b$ of a bilinear form $b: A \times A \rightarrow R$ is defined by $(-b)(x, y)=-b(x, y)$. A quadratic form $q$ is said to be over a bilinear form $b: A \times A \rightarrow R$ if $b_{q}=b$. A bilinear form $b: A \times A \rightarrow R$ gives rise to a quadratic form $q_{b}: A \rightarrow R$ by $q_{b}(x)=b(x, x)$. The following relations hold between the forms $q_{b}$ and $b_{q}: q_{b_{q}}(x)=2 q(x)$ and $b_{q_{b}}(x, y)=b(x, y)+b(y, x)$.

We recall the notion of hyperbolic form. Given a finite abelian group $G$, we define its dual by $G^{*}=\operatorname{Hom}(G, \mathbf{Q} / \mathbf{Z})$. We say that a symmetric bilinear form $b: G \times G \rightarrow \mathbf{Q} / \mathbf{Z}$ is hyperbolic if it is isomorphic to $b_{H}: H \times H \rightarrow \mathbf{Q} / \mathbf{Z}$ with $H=M \oplus M^{*}$ where $M$ is a finite abelian group and

$$
b_{H}((x, \alpha),(y, \beta))=\alpha(y)+\beta(x), \quad x, y \in M, \alpha, \beta \in M^{*} .
$$

We say that a quadratic form $q: G \rightarrow \mathbf{Q} / \mathbf{Z}$ is hyperbolic if it is isomorphic to $q_{H}: H \rightarrow \mathbf{Q} / \mathbf{Z}$ with $H=M \oplus M^{*}$ where $M$ is a finite abelian group and

$$
q_{H}(x, \alpha)=\alpha(x), \quad x \in M, \alpha \in M^{*} .
$$

Note that if a quadratic form $q$ is hyperbolic, then its associated form is also hyperbolic.

Given a quadratic form $q: G \rightarrow \mathbf{Q} / \mathbf{Z}$ and an integral symmetric bilinear form $f: F \times F \rightarrow \mathbf{Z}$ on a lattice, there is a unique quadratic form $q \otimes f: G \otimes F \rightarrow \mathbf{Q} / \mathbf{Z}$ such that $(q \otimes f)(x \otimes y)=q(x) f(y, y)$ for all $x \in G$ and $y \in F$. Cf. [Fr], [Sa]. In general, the tensor product of non-singular forms gives rise to pairings of Witt groups. However, the product of a non-degenerate quadratic form and a nondegenerate symmetric bilinear form need not be non-degenerate. (For example, take $q: \mathbf{Z} / 2 \mathbf{Z} \rightarrow \mathbf{Q} / \mathbf{Z}, 1 \mapsto \frac{1}{4}$ and the symmetric bilinear form on $\mathbf{Z}$ which maps $(1,1)$ to 2 .)

We now fix the notation which we use throughout the rest of the paper. For the applications we have in mind, it is convenient to introduce the following monoids (for direct sum):

\footnotetext{
${ }^{1}$ A symmetric bilinear form $b: A \times A \rightarrow R$ is said to be non-degenerate (resp. non-singular) if its adjoint homomorphism ad $b: A \rightarrow \operatorname{Hom}_{R}(A, R)$ is injective (resp. is an isomorphism).
} 
- $\mathfrak{M}_{\mathbf{Z}}$ denotes the monoid of (isomorphism classes of) pairs $(F, f)$ where $f$ : $F \times F \rightarrow \mathbf{Z}$ is a symmetric bilinear form on a lattice $F$.

- $\mathfrak{M}_{\mathbf{Z}}^{\mathrm{Wu}}$ denotes the monoid whose elements are (isomorphism classes of) triples (a lattice $F$, a symmetric bilinear form on $F$, a Wu class considered modulo $2 F$ for this form).

- $\mathfrak{M}$ denotes the monoid of (isomorphism classes of) pairs $(G, b)$ where $b$ : $G \times G \rightarrow \mathbf{Q} / \mathbf{Z}$ is a non-degenerate symmetric bilinear form on a finite abelian group $G$.

. $\mathfrak{M Q}$ denotes the monoid (for direct sum) of isomorphism classes of pairs $(G, q)$ where $q: G \rightarrow \mathbf{Q} / \mathbf{Z}$ is a non-degenerate quadratic form on a finite abelian group $G$.

$\widehat{\mathfrak{M}}$ is the monoid of equivalence classes of $\mathfrak{M}$ for the following equivalence relation: $(G, b),\left(G^{\prime}, b^{\prime}\right) \in \mathfrak{M}$ are equivalent if there exist hyperbolic symmetric bilinear forms $b_{1}: G_{1} \times G_{1} \rightarrow \mathbf{Q} / \mathbf{Z}$ and $b_{2}: G_{2} \times G_{2} \rightarrow \mathbf{Q} / \mathbf{Z}$ such that $(G, b) \oplus\left(G_{1}, b_{1}\right)=\left(G^{\prime}, b^{\prime}\right) \oplus\left(G_{2}, b_{2}\right)$ in $\mathfrak{M}$.

. $\widehat{\mathfrak{Q Q}}$ is the monoid of equivalence classes of $\mathfrak{M Q}$ for the following equivalence relation: $(G, q),\left(G^{\prime}, q^{\prime}\right) \in \mathfrak{M Q}$ are equivalent if there exist hyperbolic quadratic forms $q_{1}: G_{1} \rightarrow \mathbf{Q} / \mathbf{Z}$ and $q_{2}: G_{2} \rightarrow \mathbf{Q} / \mathbf{Z}$ such that $(G, q) \oplus\left(G_{1}, q_{1}\right)=\left(G^{\prime}, q^{\prime}\right) \oplus\left(G_{2}, q_{2}\right)$ in $\mathfrak{M Q}$.

The monoids introduced above fit in the following (non exact) sequences of maps:

$$
\begin{gathered}
\mathfrak{M}_{\mathbf{Z}} \stackrel{L}{\rightarrow} \mathfrak{M} \stackrel{\text { proj }}{\rightarrow} \widehat{\mathfrak{M}}, \\
\mathfrak{M}_{\mathbf{Z}}^{\text {Wu }} \stackrel{\phi}{\rightarrow} \mathfrak{M Q} \stackrel{\text { proj }}{\rightarrow} \widehat{\mathfrak{M Q}} .
\end{gathered}
$$

Here $L$ and $\phi$ are the maps defined by (10) and (12) respectively (see $\S 1.2$ ).

For a nonzero integer $m$, we denote by $(m)$ the unique bilinear form on $\mathbf{Z}$ sending $(1,1)$ to $m$. Let $a$ and $b$ be coprime integers such that $0<|a|<b$. We denote by $\left(\frac{a}{b}\right)$ the unique bilinear form on $\mathbf{Z} / b \mathbf{Z}$ sending $(1,1)$ to $\frac{a}{b} \in \mathbf{Q} / \mathbf{Z}$. We denote by $E_{0}^{k}$ $(1 \leq k)$ and $E_{1}^{k}(2 \leq k)$ the bilinear forms on $\mathbf{Z} / 2^{k} \mathbf{Z} \oplus \mathbf{Z} / 2^{k} \mathbf{Z}$ determined by the matrices

$$
\left(\begin{array}{cc}
0 & 2^{-k} \\
2^{-k} & 0
\end{array}\right) \text { and }\left(\begin{array}{cc}
2^{1-k} & 2^{-k} \\
2^{-k} & 2^{1-k}
\end{array}\right)
$$

respectively. Notice that all these forms are non-degenerate and $E_{0}^{k}$ is hyperbolic. These notations agree with those of $[\mathrm{KK}]$ and $[\mathrm{Mu}]$.

2.2. The correspondence from integral forms to forms over finite abelian groups. We need to formulate the main result about the correspondence discussed in $\S 1.2$. This result will be instrumental in the proof of Theorem 1.

In $\mathfrak{M}_{\mathbf{Z}}$, we consider the equivalence relation, denoted by $\sim$, generated by the following operation: $(F, f) \mapsto(F \oplus \mathbf{Z}, f \oplus( \pm 1))$.

In $\mathfrak{M}_{\mathbf{Z}}^{\mathrm{Wu}}$, we define the equivalence relation, also denoted by $\sim$, generated by the following operation: $(F, f, w) \mapsto\left(F \oplus \mathbf{Z}, f \oplus( \pm 1), w \oplus w^{\prime}\right)$ where $w^{\prime}$ is an odd integer.

Lemma 2.1. (a) The homomorphism $\mathfrak{M}_{\mathbf{Z}} \rightarrow \mathfrak{M},(F, f) \mapsto\left(K_{f}, L_{f}\right)$ is surjective. For $\left(F^{\prime}, f^{\prime}\right) \in \mathfrak{M}_{\mathbf{Z}}$, the following two conditions are equivalent: 
(a.2) $\operatorname{ker}$ ad $f \cong \operatorname{ker}$ ad $f^{\prime}$ and $\left(\right.$ Tors $(\operatorname{coker}$ ad $\left.f), L_{f}\right) \cong\left(\right.$ Tors $\left(\right.$ coker ad $\left.\left.f^{\prime}\right), L_{f^{\prime}}\right)$.

(b) The homomorphism $\mathfrak{M}_{\mathbf{Z}}^{W u} \rightarrow \mathfrak{M Q},(F, f, w) \mapsto\left(K_{f}, \phi_{f, w}\right)$ is surjective. For $(F, f, w),\left(F^{\prime}, f^{\prime}, w^{\prime}\right) \in \mathfrak{M}_{\mathbf{Z}}^{W u}$, the following two conditions are equivalent:

(b.1) $(F, f, w) \sim\left(F^{\prime}, f^{\prime}, w^{\prime}\right)$;

(b.2) $\operatorname{ker}$ ad $f \cong \operatorname{ker}$ ad $f^{\prime}$ and $\left(\right.$ Tors $(\operatorname{coker}$ ad $\left.f), \phi_{f, w}\right) \cong\left(\right.$ Tors $\left.\left(\operatorname{coker} \operatorname{ad} f^{\prime}\right), \phi_{f^{\prime}, w^{\prime}}\right)$.

Proof. Denote by $\mathfrak{M}_{\mathbf{Z}}^{0}$ the monoid which consists of isomorphism classes of pairs $(F, f)$ where $f: F \times F \rightarrow \mathbf{Z}$ is an even symmetric bilinear form on a lattice $F$. The surjectivity of the maps $\mathfrak{M}_{\mathbf{Z}} \rightarrow \mathfrak{M},(F, f) \mapsto\left(K_{f}, L_{f}\right)$ and $\mathfrak{M}_{\mathbf{Z}}^{0} \rightarrow \mathfrak{M Q},(F, f) \mapsto$ $\left(K_{f}, \phi_{f}\right)$ was proved by C.T.C. Wall [Wa, Theorem 6]. See also [Du, Theorems 4.4 and 4.7] and [La] for generalizations. The surjectivity of $\mathfrak{M}_{\mathbf{Z}}^{\mathrm{Wu}} \rightarrow \mathfrak{M Q Q},(F, f, w) \mapsto$ $\left(K_{f}, \phi_{f, w}\right)$ is a direct consequence of the surjectivity of $\mathfrak{M}_{\mathbf{Z}}^{0} \rightarrow \mathfrak{M Q},(F, f) \mapsto$ $\left(K_{f}, \phi_{f}\right)$ since $\mathfrak{M}_{\mathbf{Z}}^{0} \subset \mathfrak{M}_{\mathbf{Z}}^{\mathrm{Wu}}$ (for $f$ even, $\left.\phi_{f}=\phi_{f, 0}\right)$. The implications (a.1) $\Longrightarrow$ (a.2) and (b.1) $\Longrightarrow$ (b.2) are straightforward. The converse (a.2) $\Longrightarrow$ (a.1) can be found in [Du, Corollary 4.2], in the case when $f$ and $g$ are non-degenerate, but the argument given applies in our case as well: simply decompose $f$ (resp. $g$ ) as a direct sum of a 0 -form and of a non-degenerate form on a summand of the lattice $F$ (resp. of the lattice $F^{\prime}$ ). For the implication (b.2) $\Longrightarrow$ (b.1), note that, since $\phi_{f, w}$ is a quadratic form over $L_{f}$, there is an isomorphism (Tors (coker ad $\left.\left.f\right), L_{f}\right) \cong$ (Tors $\left(\right.$ coker ad $\left.\left.f^{\prime}\right), L_{f^{\prime}}\right)$. Applying (a), we obtain that $(F, f) \sim\left(F^{\prime}, f^{\prime}\right)$. We can assume that $k=\operatorname{rank} F^{\prime}-\operatorname{rank} F \geq 0$. Thus there exist $k$ integers $v_{1}, \ldots, v_{k}$ such that $w^{\prime}=w \oplus \bigoplus_{j=1}^{k} v_{j}$. It follows from the definition of $\phi_{f, w}$ that $v_{j} \equiv 1 \bmod 2$ for $j=1, \ldots, k$. This is the desired result.

The importance of the constructions described in $\S 1.2$ and lemma 2.1 in algebraic topology lies in the following fact. Let $B_{W}: H_{2}(W ; \mathbf{Z}) \times H_{2}(W ; \mathbf{Z}) \rightarrow \mathbf{Z}$ be the intersection form of a compact simply connected 4-manifold, let $M=\partial W$ and let $\mathcal{L}_{M}$ : Tors $H_{1}(M ; \mathbf{Z}) \times$ Tors $H_{1}(M ; \mathbf{Z}) \rightarrow \mathbf{Q} / \mathbf{Z}$ be the linking form of $M$. Then

$$
\left(K_{B_{W}},-L_{B_{W}}\right)=\left(\text { Tors } H_{1}(M ; \mathbf{Z}), \mathcal{L}_{M}\right) .
$$

Furthermore, even though we will not use it, we recall the following fact: $M$ always admits a spin structure (see [Ki2] for example) and it is known (see [Rok]) that this spin structure can be extended to the 4-manifold $W$; in this case, $B_{W}$ is even and the form $\phi_{B_{W}}$ defined by (11) is a quadratic form over $L_{B_{W}}=-\mathcal{L}_{M}$ and depends only on the spin structure on $M[\mathrm{Tu} 2]$.

\subsection{Elementary properties of Gauss sums.}

Lemma 2.2. Let $f: G \rightarrow \mathbf{Q} / \mathbf{Z}$ be a homomorphism where $G$ is a finite group. Then

$$
\sum_{\alpha \in G} e^{2 \pi i f(\alpha)}= \begin{cases}|G| & \text { if } f=0, \\ 0 & \text { otherwise. }\end{cases}
$$

An application of lemma 2.2 leads to

Lemma 2.3. Let $G, H$ be finite abelian groups and $f$ be a bilinear pairing $G \times H \rightarrow$ $\mathbf{Q} / \mathbf{Z}$. Let ad $f: H \rightarrow \operatorname{Hom}(G, \mathbf{Q} / \mathbf{Z})$ be the left adjoint homomorphism. For any $\beta \in H$,

$$
\sum_{\alpha \in G} e^{2 \pi i f(\alpha, \beta)}= \begin{cases}|G| & \text { if } \beta \in \operatorname{ker} \text { ad } f \\ 0 & \text { otherwise }\end{cases}
$$


Corollary. Let $G$ be a finite abelian group and $G^{*}=\operatorname{Hom}(G, \mathbf{Q} / \mathbf{Z})$. For any bilinear pairing $f: G \times G^{*} \rightarrow \mathbf{Q} / \mathbf{Z}$, the sum

$$
\sum_{(x, \alpha) \in G \times G^{*}} e^{2 \pi i f(x, \alpha)}
$$

is a positive real number.

We now proceed to the proof of the lemma 1.1 preliminary to the definition of the invariant $\tau(M ; G, q)$ in $\S 1.1$.

Proof of lemma 1.1. We rewrite $\left|\sum_{g \in G} e^{2 \pi i q(g)}\right|^{2}$ as

$\sum_{g \in G} e^{2 \pi i q(g)} \sum_{h \in G} \overline{e^{2 \pi i q(h)}}=\sum_{g \in G} e^{2 \pi i q(g)} \sum_{h \in G} e^{-2 \pi i q(h)}=\sum_{g \in G}\left(\sum_{h \in G} e^{2 \pi i b_{q}(g, h)}\right) e^{2 \pi i q(g)}$.

Applying lemma 2.3, we obtain

$$
\left|\sum_{g \in G} e^{2 \pi i q(g)}\right|^{2}=|G| \sum_{g \in \operatorname{ker~ad} b_{q}} e^{2 \pi i q(g)} .
$$

We observe that the restriction of $q$ to $\operatorname{ker} \operatorname{ad} b_{q}$ is a homomorphism $\operatorname{ker} \operatorname{ad} b_{q} \rightarrow$ $\{1,-1\} \cong \mathbf{Z} / 2 \mathbf{Z}$. Consequently,

$$
\sum_{g \in \text { ker ad } b_{q}} q(g)= \begin{cases}\left|\operatorname{ker} \operatorname{ad} b_{q}\right| & \text { if } q\left(\operatorname{ker} \operatorname{ad} b_{q}\right)=0, \\ 0 & \text { otherwise. }\end{cases}
$$

The proof is complete.

We need to make the condition $q\left(\operatorname{ker} \operatorname{ad} b_{q}\right)=0$ more explicit. This is the purpose of the next lemma.

Lemma 2.4. Let $q$ be a quadratic form $G \rightarrow \mathbf{Q} / \mathbf{Z}$ on a finite abelian group $G$. The following assertions are equivalent:

(1) $q\left(\right.$ ker ad $\left.b_{q}\right)=0$;

(2) $q(H)=0$ for any 2-cyclic summand $H$ of $G$ which lies in $\operatorname{ker}$ ad $b_{q}$.

Proof. The implication (1) $\Longrightarrow(2)$ is obvious. We show the implication $(2) \Longrightarrow(1)$. For any $g \in G, 2 q(g)=b_{q}(g, g)$. If $|G|$ is odd, then $2 q(g)=0$ implies $q(g)=0$ (since the order of $q(g)$ in $\mathbf{Q} / \mathbf{Z}$ must be odd $)$. It follows that $q\left(\operatorname{ker} a d b_{q}\right)=0$. Assume $|G|$ to be even. There is an orthogonal splitting $(G, q)=\bigoplus_{p}\left(G_{p}, q_{p}\right)$ where $p$ runs over prime numbers, $G_{p}$ is a $p$-subgroup of $G, G=\bigoplus_{p} G_{p}$ and $q_{p}=\left.q\right|_{G_{p}}$. Therefore we may assume that $G$ itself is a (finite abelian) 2-group. Let $x \in \operatorname{ker}$ ad $b_{q}$ and let $H$ be the cyclic subgroup of $G$ generated by $x$. Its order is a power of 2 . By definition of $x, H$ is orthogonal to $G$. If $H$ is a summand of $G$, then condition (2) applies, so that $\left.q\right|_{H}=0$ and hence $q(x)=0$. If $H$ is not a summand of $G$, then $H \subset 2 G$. Therefore there exists an element $y \in G$ such that $x=2 y$. Then $q(x)=q(2 y)=4 q(y)=2 b_{q}(y, y)=b_{q}(2 y, y)=b_{q}(x, y)=\operatorname{ad} b_{q}(x)(y)=0$.

Remarks. 1. The proof shows that a sufficient, but not necessary, assumption to ensure condition (1) of lemma 2.4 is $\operatorname{ker}$ ad $b_{q} \subset 2 G$.

2. From lemma 2.4, one deduces the following condition: $q\left(\operatorname{ker} \operatorname{ad} b_{q}\right)=0$ if and only if there exists a 2-cyclic summand $H$ of $G$ which lies in ker ad $b_{q}$ such that $\left.q\right|_{H}(x)=\frac{1}{2}$ if $x$ generates $H,\left.q\right|_{H}(x)=0$ otherwise. 
The next two lemmas are preparation for the proof of lemma 1.2.

Let $G$ be a finite abelian group and $q: G \rightarrow \mathbf{Q} / \mathbf{Z}$ be a quadratic form on $G$. Set $K=\operatorname{ker} \operatorname{ad} b_{q}$ and $\tilde{G}=G / K$.

Lemma 2.5. The following relation holds:

$$
\sum_{x \in G} e^{2 \pi i q(x)}= \begin{cases}0 & \text { if } q(K) \neq 0, \\ \left|\operatorname{ker} \operatorname{ad} b_{q}\right| \sum_{x \in \tilde{G}} e^{2 \pi i \tilde{q}(x)} & \text { if } q(K)=0,\end{cases}
$$

where $\tilde{q}: \tilde{G} \rightarrow \mathbf{Q} / \mathbf{Z}$ is the quadratic form induced by $q$.

Proof. If $q(K) \neq 0$, then the result follows from lemma 1.1. If $q(K)=0$, then it is clear that $q: G \rightarrow \mathbf{Q} / \mathbf{Z}$ induces a non-degenerate quadratic form $\tilde{q}: \tilde{G} \rightarrow \mathbf{Q} / \mathbf{Z}$. The result follows easily.

Lemma 2.6. Let $B: F \times F \rightarrow \mathbf{Z}$ be a symmetric bilinear form on a lattice $F$. Then

$$
\sum_{x \in G \otimes F} e^{2 \pi i(q \otimes B)(x)}= \begin{cases}0 & \text { if }(q \otimes B)(K \otimes F) \neq 0, \\ |K \otimes F| \sum_{x \in \tilde{G} \otimes F} e^{2 \pi i(\widetilde{q \otimes B})(x)} & \text { if }(q \otimes B)(K \otimes F)=0,\end{cases}
$$

where $\widetilde{q \otimes B}=\tilde{q} \otimes B: \tilde{G} \otimes F \rightarrow \mathbf{Q} / \mathbf{Z}$ is the quadratic form induced by $q \otimes B$.

Proof. Analogous to the proof of the previous lemma. The key observation is that $K \otimes F \subset \operatorname{ker}\left(\operatorname{ad} b_{q} \otimes \operatorname{ad} B\right)$.

Proof of lemma 1.2. We keep the same notation as in the two lemmas above. Since $\gamma(G, q) \neq 0$, lemma 1.1 ensures that $q(K)=0$. Lemma 2.5 applies and yields

$$
\gamma(G, q)=\gamma(\tilde{G}, \tilde{q}) .
$$

Next, $q(K)=0$ implies $\left(q \otimes B_{W}\right)\left(K \otimes H_{2}(W ; \mathbf{Z})\right)=0$. So lemma (2.6) applies:

$$
\sum_{x \in G \otimes H_{2}(W ; \mathbf{Z})} e^{2 \pi i\left(q \otimes B_{W}\right)(x)}=\left|K \otimes H_{2}(W ; \mathbf{Z})\right| \cdot \sum_{x \in \tilde{G} \otimes H_{2}(W ; \mathbf{Z})} e^{2 \pi i\left(\widetilde{\otimes \otimes B_{W}}\right)(x)} .
$$

Comparing equations (18) and (19) with the definition (2) of $\tau(M ; G, q)$, we obtain the desired result.

The following two lemmas (2.7 and 2.8) will be useful in proving Theorems 1, 3 and 5 . We denote by $\mu_{8}$ the group of complex 8 -th roots of unity.

Lemma 2.7. Let $f: F \rightarrow \mathbf{Z}$ be a symmetric bilinear form on a lattice. Let $q: G \rightarrow$ $\mathbf{Q} / \mathbf{Z}$ be a quadratic form on a finite abelian group. The map $\mathfrak{M Q} \rightarrow \mathbf{C},(G, q) \mapsto$ $\gamma(G \otimes F, q \otimes f)$ induces a homomorphism $\widehat{\mathfrak{M Q}} \rightarrow \mu_{8} \cup\{0\}$.

Proof. For multiplicativity of Gauss sums and the fact that the image is in $\mu_{8} \cup\{0\}$, see for example [Sc, chapter 5]. It suffices to show that $\gamma(G \otimes V, q \otimes f)=1$ for $q$ hyperbolic. Suppose $G=M \oplus M^{*}$ where $M$ is a finite abelian group and * denotes usual duality, i.e. $(.)^{*}=\operatorname{Hom}(., \mathbf{Q} / \mathbf{Z})$ and $q(x, \nu)=\nu(x)$. Choose a base $\left(e_{1}, \ldots, e_{m}\right)$ for $F$. Then $q \otimes f$ can be viewed as a quadratic form

$$
G \otimes F=M^{m} \oplus\left(M^{m}\right)^{*} \rightarrow \mathbf{Q} / \mathbf{Z}, \quad(\mathbf{x}, \nu) \mapsto \sum_{i, j} f_{i j} \nu_{j}\left(x_{i}\right)
$$


where $\left(f_{i j}\right)_{1 \leq i, j \leq n}$ is the matrix of $f, \mathbf{x}=\left(x_{1}, \ldots, x_{m}\right)$ and $\nu=\left(\nu_{1}, \ldots, \nu_{m}\right)$. Observe that the map

$$
M^{m} \times\left(M^{m}\right)^{*} \rightarrow \mathbf{Q} / \mathbf{Z}, \quad(\mathbf{x}, \nu) \mapsto \sum_{i, j} f_{i j} \nu_{j}\left(x_{i}\right)
$$

is a bilinear pairing. Therefore it follows from the corollary of lemma 2.3 that

$$
\sum_{x \in M^{m} \times\left(M^{m}\right)^{*}} e^{2 \pi i(q \otimes f)(x)}
$$

is a nonzero real number. Since $\gamma(G \otimes F, q \otimes f) \in \mu_{8} \cup\{0\}$ (or by lemma 1.1), we deduce that $\gamma(G \otimes F, q \otimes f)=1$.

Lemma 2.8. Let $f: F \rightarrow \mathbf{Z}$ be a symmetric bilinear form on a finitely generated abelian group. Let $q: G \rightarrow \mathbf{Q} / \mathbf{Z}$ be a quadratic form on a finite abelian group. Let $B: \mathfrak{M}_{\mathbf{Z}} \rightarrow \mu_{8} \cup\{0\}$ be the map defined by

$$
(F, f) \mapsto \overline{\gamma(G, q)}^{\sigma(f)} \gamma(G \otimes F, q \otimes f) .
$$

It induces a homomorphism $\tilde{B}: \widehat{\mathfrak{M}} \rightarrow \mu_{8} \cup\{0\}$ making the following diagram commute:

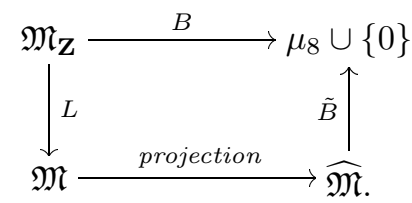

Proof. To simplify notation, we write $B(f)$ instead of $B(F, f)$. The multiplicativity of $B$ is clear. First, we show that $B(f)$ only depends on (the isomorphism class of) $L_{f}$. Observe that

$$
\begin{aligned}
B(f \oplus( \pm 1)) & =\overline{\gamma(G, q)}^{\sigma(f \oplus( \pm 1))} \gamma(G \otimes(F \oplus \mathbf{Z}), q \otimes(f \oplus( \pm 1))) \\
& =\overline{\gamma(G, q)}^{\sigma(f)} \gamma(G \otimes F, q \otimes f) \overline{\gamma(G, q)}^{ \pm 1} \gamma(G, \pm q) \\
& =B(f) .
\end{aligned}
$$

If $f$ and $f^{\prime}$ are isomorphic forms, it is clear that $B(f)=B\left(f^{\prime}\right)$. By lemma 2.1, $(a)$, it follows that $B$ only depends on the isomorphism class of $L_{f}$ and ker ad $f$. We prove that $B$ does not depend on ker ad $f$. Let $f: F^{\prime} \times F^{\prime} \rightarrow \mathbf{Z}$ be a symmetric bilinear form on a finitely generated abelian group such that $L_{f^{\prime}}=L_{f}$. We can assume $k=\operatorname{rank}\left(\operatorname{ker} a d f^{\prime}\right)-\operatorname{rank}(\operatorname{ker} \operatorname{ad} f) \geq 0$. Consider the symmetric bilinear form $\tilde{f}$ on $\tilde{F}=F \oplus\left(\bigoplus_{j=1}^{k} \mathbf{Z}\right)$ defined by

$$
(\tilde{F}, \tilde{f})=(F, f) \oplus \bigoplus_{j=1}^{k}(\mathbf{Z}, 0) .
$$

It is easy to see that $\left(K_{\tilde{f}}, L_{\tilde{f}}\right)=\left(K_{f}, L_{f}\right)=\left(K_{f^{\prime}}, L_{f^{\prime}}\right)$. Furthermore, $\operatorname{rank}(\operatorname{ker} \operatorname{ad} \tilde{f})=\operatorname{rank}(\operatorname{ker} \operatorname{ad} f)+k=\operatorname{rank}\left(\operatorname{ker} a d f^{\prime}\right)$.

We deduce that $B(\tilde{f})=B\left(f^{\prime}\right)$. The multiplicativity of $B$ yields

$$
B(\tilde{f})=B(f) \cdot \prod_{j=1}^{k} B(0)=B(f)
$$


since $B(0)=B(\mathbf{Z}, 0)=1$. Therefore, $B\left(f^{\prime}\right)=B(f)$, which is the claimed property. To conclude, it suffices to show that $B(f)=1$ for $L_{f}$ hyperbolic. The canonical decomposition of $K_{f}$ in $p$-primary components is orthogonal with respect to $L_{f}$. Moreover, the property of being hyperbolic is preserved by restriction on each $p$ primary component. Since by lemma 2.1 the map $(F, f) \mapsto\left(K_{f}, L_{f}\right)$ is a surjective homomorphism, we can assume that $\left(K_{f}, L_{f}\right)$ is irreducible. In particular, it is a bilinear pairing on a (finite abelian) $p$-group, isomorphic, for some prime $p$ and positive integer $m$, to the bilinear pairing on $\mathbf{Z} / p^{m} \mathbf{Z} \times \mathbf{Z} / p^{m} \mathbf{Z}$ determined by the matrix

$$
\left(\begin{array}{cc}
0 & p^{-k} \\
p^{-k} & 0
\end{array}\right)
$$

We can choose $f$ to be the bilinear form on $\mathbf{Z}^{2}$ determined by the matrix

$$
\left(\begin{array}{cc}
0 & p^{k} \\
p^{k} & 0
\end{array}\right)
$$

Thus $q \otimes f$ can be viewed as the quadratic form

$$
G \oplus G \rightarrow \mathbf{Q} / \mathbf{Z},(x, y) \mapsto p^{k} b_{q}(x, y) .
$$

We observe that the map

$$
G \times G \rightarrow \mathbf{Q} / \mathbf{Z},(x, y) \mapsto p^{k} b_{q}(x, y)
$$

is a bilinear pairing. Therefore, from the corollary of lemma 2.3 , it follows that

$$
\sum_{x \in G \oplus G} e^{2 \pi i(q \otimes f)(x)}
$$

is a positive real number. Lemma 1.1 implies that $\gamma\left(G \otimes \mathbf{Z}^{2}, q \otimes f\right)=1$. Since $\sigma(f)=0$, the result follows.

\section{Properties of $\tau(M ; G, q)$}

By lemma 1.2, we can assume $q$ to be non-degenerate $\left(\operatorname{ker} a d b_{q}=0\right)$.

3.1. Proof of Theorem 1. Since the expression defining $\tau(M ; G, q)$ depends on the intersection form $B_{W}$, we write temporarily $\tau\left(B_{W} ; G, q\right)$ throughout this paragraph. Set $H_{2}=H_{2}(W ; \mathbf{Z})$. We first prove the first statement in the theorem. Recall that ker ad $B_{W} \cong H^{1}(M ; \mathbf{Z})$ (cf. lemma 3.2). By means of the lemma 2.1, (a), and the remark we made thereafter, it suffices to show invariance of $\tau\left(B_{W} ; G, q\right)$ on the equivalence class of $\left(H_{2}, B_{W}\right)$ in $\mathfrak{M}_{\mathbf{Z}}$. The change of the form $B_{W}$ into an isomorphic form clearly does not affect the expression. If $\left(H_{2}, B_{W}\right)$ is changed into $\left(H_{2} \oplus \mathbf{Z}, B_{W} \oplus( \pm 1)\right)$, then

$$
\begin{gathered}
\tau\left(B_{W} \oplus( \pm 1) ; G, q\right) \\
=\overline{\gamma(G, q)}{ }^{\sigma\left(B_{W}\right) \pm 1}\left|G \otimes H_{2}\right|^{-\frac{1}{2}}|G|^{-\frac{1}{2}} \sum_{x \in G \otimes H_{2}} e^{2 \pi i\left(q \otimes B_{W}\right)(x)} \sum_{x \in G} e^{ \pm 2 \pi i q(x)} .
\end{gathered}
$$

It follows from the definition of $\gamma(G, q)$ that $\tau\left(B_{W} \oplus( \pm 1) ; G, q\right)=\tau\left(B_{W} ; G, q\right)$. This is the desired result.

To prove the second statement, we observe that the phase of $\tau(M ; G, q)$ is exactly $\overline{\gamma(G, q)}{ }^{\sigma\left(B_{W}\right)} \gamma\left(G \otimes H_{2}, q \otimes B_{W}\right)$. Therefore, the result follows from lemma 2.8. 
3.2. Proof of Theorem 2. We begin with some elementary algebraic lemmas.

Lemma 3.1. Let $F$ be a lattice and $B: F \times F \rightarrow \mathbf{Z}$ be a symmetric bilinear form. Let $g: G \times G \rightarrow \mathbf{Q} / \mathbf{Z}$ be a non-degenerate symmetric bilinear form on an abelian group $G$. Then

$$
\operatorname{ker}(\operatorname{ad} g \otimes \operatorname{ad} B)=\operatorname{ker}\left(\operatorname{id}_{G} \otimes \operatorname{ad} B\right) .
$$

Proof. There is an obvious commutative diagram:

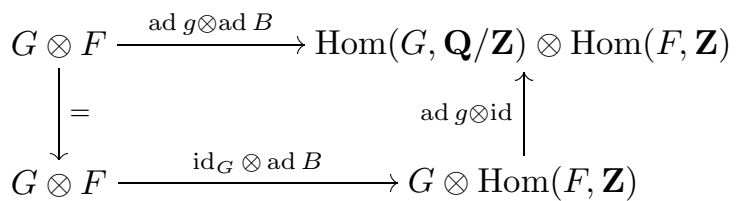

The homomorphism ad $g: G \rightarrow \operatorname{Hom}(G, \mathbf{Q} / \mathbf{Z})$ is an isomorphism. Since, as a $\mathbf{Z}$ module, $F$ is free (hence flat), the map ad $g \otimes$ id is also an isomorphism. Hence $\operatorname{ker}(\operatorname{ad} g \otimes \operatorname{ad} B)=\operatorname{ker}\left(\operatorname{id}_{G} \otimes \operatorname{ad} B\right)$.

Lemma 3.2. Let $W$ be a simply connected oriented 4-manifold such that $\partial W=M$. Let $B_{W}: H_{2}(W ; \mathbf{Z}) \times H_{2}(W ; \mathbf{Z}) \rightarrow \mathbf{Z}$ be the intersection form on $W$. Then for any abelian group $G$,

$\operatorname{ker}\left(\operatorname{id}_{G} \otimes \operatorname{ad} B_{W}\right) \cong H^{1}(M ; G)$ and $\operatorname{coker}\left(\operatorname{id}_{G} \otimes \operatorname{ad} B_{W}\right) \cong H_{1}(M ; G)$.

Proof. This follows from the Poincaré duality and the homological sequence of the pair $(W, \partial W)$ with coefficients in $G$.

Lemma 3.3. The following relation holds for an arbitrary non-degenerate quadratic form $q: G \rightarrow \mathbf{Q} / \mathbf{Z}$ on a finite abelian group $G$ :

$$
\begin{gathered}
\left|\sum_{x \in G \otimes H_{2}(W ; \mathbf{Z})} \exp \left(2 \pi i\left(q \otimes B_{W}\right)(x)\right)\right|^{2} \\
=\left\{\begin{array}{cl}
\left|G \otimes H_{2}(W ; \mathbf{Z})\right|\left|H^{1}(M ; G)\right| & \text { if }\left(q \otimes B_{W}\right)\left(\operatorname{ker}\left(\operatorname{ad} b_{q} \otimes \operatorname{ad} B_{W}\right)\right)=0, \\
0 & \text { otherwise. }
\end{array}\right.
\end{gathered}
$$

Proof. Apply lemma 1.1 to the finite abelian group $G \otimes H_{2}(W ; \mathbf{Z})$ equipped with the quadratic form $q \otimes B_{W}$. The bilinear form $b_{q \otimes B_{W}}$ associated to $q \otimes B_{W}$ is equal to $b_{q} \otimes B_{W}$. So $\left|\operatorname{ker} \operatorname{ad} b_{q \otimes B_{W}}\right|=\left|\operatorname{ker}\left(\operatorname{ad} b_{q} \otimes \operatorname{ad} B_{W}\right)\right|$ and the result follows from lemmas 3.1 and 3.2 .

Now Theorem 2 follows from the definition $(2)$ of $\tau(M ; G, q)$ and lemma 3.3.

3.3. The phase of $\tau(M ; G, q)$. In this section, we shall use the notations introduced in $\S 2.1$. Denote by $\mu_{8}$ the group of 8 -th roots of unity and by $T$ the finite abelian group Tors $H_{1}(M ; \mathbf{Z})$.

Consider the phase of $\tau(M ; G, q)$ :

$$
\beta_{q}\left(\mathcal{L}_{M}\right)=\frac{\tau(M ; G, q)}{\left|H^{1}(M ; G)\right|^{\frac{1}{2}}} .
$$


The following theorem shows that the invariant $\beta_{q}$ can be interpreted as an invariant of the class of $\mathcal{L}_{M}$ in $\widehat{\mathfrak{M}}$.

Theorem 5. The map $\beta$ induces a bilinear pairing

$$
\tilde{\beta}: \widehat{\mathfrak{M Q}} \times \widehat{\mathfrak{M}} \rightarrow \mu_{8} \cup\{0\},\left([G, q],\left[T, \mathcal{L}_{M}\right]\right) \mapsto \beta_{q}\left(\mathcal{L}_{M}\right)
$$

making the following diagram commute:

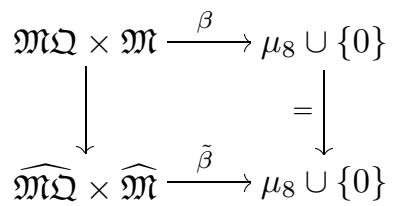

where the left vertical arrow is the canonical projection.

As a consequence of Theorem 5 , we mention the following result.

Corollary. If $|G|$ or $|T|$ is odd, then $\beta_{q}\left(\mathcal{L}_{M}\right)$ is a 4-th root of unity.

Remark. Theorem 5 and the commutative diagram above are reminiscent of the action of the Witt group $W(\mathbf{Z})$ of unimodular symmetric bilinear forms on (finitely generated) free $\mathbf{Z}$-modules on the Witt group $W Q(\mathbf{Q}, \mathbf{Z})$ of quadratic forms on finite abelian groups with values in $\mathbf{Q} / \mathbf{Z}$. See for example [La] for a careful treatment of this action (as well as its algebraic generalizations). From the topological point of view, however, if the form $B_{W}$ is unimodular, the invariant is trivial. Theorem 5 suggests that one can still define an action of (non necessarily unimodular) symmetric bilinear forms in the context of Witt monoids.

Proof of Theorem 5. As a consequence of Theorem 2, we have (cf. (7))

$$
\beta_{q}\left(\mathcal{L}_{M}\right)=\overline{\gamma(G, q)}^{\sigma\left(B_{W}\right)} \gamma\left(G \otimes H_{2}(W ; \mathbf{Z}), q \otimes B_{W}\right) .
$$

We already know, by Theorem 1 , that the right hand side of (21) only depends on $\mathcal{L}_{M}$. It follows from lemma 2.8 that for a fixed pair $(G, q)$, the homomorphism $\left(T, \mathcal{L}_{M}\right) \mapsto \beta_{q}\left(\mathcal{L}_{M}\right)$ depends only on the class $\left[T, \mathcal{L}_{M}\right] \in \widehat{\mathfrak{M}}$. This proves half of Theorem 5 . The second half (statement about $(G, q)$ ) follows from the equality $(21)$ and lemma 2.7 .

Finally, we make one observation about the phase of $\tau(M ; G, q)$. It follows from Theorems 1 and 2 that the phase of the right hand side of (7) only depends on $\mathcal{L}_{M}=-L_{B_{W}}$ and not on $B_{W}$ itself. Therefore, we can rewrite this formula without any reference to the particular choice $B_{W}$ in the preimage of $-\mathcal{L}_{M}$. We obtain the following modified version of (21):

$$
\beta_{q}\left(\mathcal{L}_{M}\right)=\overline{\gamma(G, q)}^{\sigma(B)} \gamma(G \otimes F, q \otimes B),
$$

where $B: F \times F \rightarrow \mathbf{Z}$ is any symmetric bilinear form on the finite abelian group $F$ such that $\left(K_{B},-L_{B}\right) \cong\left(\right.$ Tors $\left.H_{1}(M ; \mathbf{Z}), \mathcal{L}_{M}\right)$.

3.4. A condition of nullity for $\tau(M ; G, q)$. In this section, we give a necessary and sufficient condition for $\tau(M ; G, q)$ to vanish. Given two symmetric bilinear pairings $(G, b)$ and $\left(G^{\prime}, b^{\prime}\right)$ on finite abelian groups, we shall say that they have an isomorphic orthogonal summand if there exists orthogonal summands $H, H^{\prime}$ of $G$ and $G^{\prime}$ respectively such that $H \cong H^{\prime}$ as groups. 
Theorem 6. The following conditions are equivalent:

(1) $\tau(M ; G, q)=0$;

(2) $\left(G, b_{q}\right)$ and $\left(T, \mathcal{L}_{M}\right)$ have an isomorphic 2-cyclic orthogonal summand;

(3) There exists a 2-cyclic orthogonal summand $K$ of $(G, q)$ and a cohomology class $\alpha \in H^{1}(M ; K)$ such that $\alpha \cup \alpha \cup \alpha \neq 0$ (here $\cup$ denotes the cup product in cohomology of $M$ with coefficients in the ring $K)$.

Remark. Theorem 6 accounts for our introduction of Witt monoids (instead of Witt groups). Suppose that $\mathcal{L}_{M}$ is the metabolic form $\left(1 / 2^{k}\right) \oplus\left(-1 / 2^{k}\right)$. Then by Theorem $6, \tau(M ; G, q)$ is zero if (and only if) there is an orthogonal splitting $(G, q) \cong\left(\mathbf{Z} / 2^{k} \mathbf{Z}, q_{1}\right) \oplus\left(G^{\prime}, q^{\prime}\right)$. Since the cases when $\tau(M ; G, q)$ is zero are topologically significant, we cannot rule out metabolic forms; whereas if $\mathcal{L}_{M}$ is hyperbolic, then by Theorem $5, \beta_{G, q}(M)=1$ for any non-degenerate quadratic form $q$ on $G$.

Before we proceed to the proof of Theorem 6, we need one algebraic lemma.

Lemma 3.4. Assume that $(G, q)$ is non-degenerate. Then $\gamma(G \otimes V, q \otimes f)=0$ if and only if $\left(G, b_{q}\right)$ and $\left(K_{f}, L_{f}\right)$ have an isomorphic 2-cyclic orthogonal summand.

Our original proof in [Del] of lemma 3.4, which relies on the classification of symmetric bilinear forms on finite abelian 2-groups (see [Wa] and [KK]), goes roughly as follows. Since the condition (1) of lemma 2.4 is always satisfied for finite abelian groups of odd order, we can assume $G$ to be a finite abelian 2-group. Using the classification in $[\mathrm{Wa}]$ or $[\mathrm{KK}]$, there are essentially three (isomorphism classes of) symmetric bilinear pairings to consider, for which it is a straightforward matter to verify lemma 3.4. The following alternative proof, which does not require any classification result, is more natural and was suggested to the author by P. Vogel.

Proof. Without loss of generality, we can assume $f$ to be non-degenerate, so that by lemma $3.1, \operatorname{ker} \operatorname{ad}\left(b_{q} \otimes f\right)=\operatorname{ker} \operatorname{ad}\left(\operatorname{id}_{G} \otimes f\right)$. Then, tensoring by $G$ the exact sequence

$$
0 \longrightarrow V \stackrel{\text { ad } f}{\longrightarrow} V^{*} \longrightarrow K_{f} \longrightarrow 0
$$

yields

$$
0 \longrightarrow \operatorname{Tor}\left(G, K_{f}\right) \longrightarrow G \otimes V \stackrel{\text { id } \otimes \text { ad } f}{\longrightarrow} G \otimes V^{*} \longrightarrow G \otimes K_{f} \longrightarrow 0
$$

which shows that $\operatorname{ker} \operatorname{ad}\left(\operatorname{id}_{G} \otimes f\right)=\operatorname{Tor}\left(G, K_{f}\right)$. Since Tor preserves direct sums and $G$ and $K_{f}$ are direct sums of cyclic groups, it follows that $\operatorname{ker} \operatorname{ad}\left(b_{q} \otimes f\right)$ is generated by elements $x \otimes y$ where $x \in G$ and $y \in V$ such that $x \otimes(\operatorname{ad} f)(y)=0$ in $G \otimes V^{*}$. The latter condition is equivalent to: $(\operatorname{ad} f)(y)$ is divisible by $n$ in $V^{*}$ where $n$ is the order of $x$ in $G$. Hence, by lemma 1.1, $\gamma(G \otimes V, q \otimes f)=0$ if and only if there exist $x$ and $y$ as above such that $(q \otimes f)(x \otimes y)=q(x) f(y, y) \neq 0$. We deduce that $q(x) f(y, y) \neq 0$ if and only if $n$ is even (here we use the fact that $q$ is non-degenerate) and $x$ generates an orthogonal summand of $G$. Denote by $\operatorname{val}_{2}(n)$ the 2-valuation of $n$. Since $\mathbf{Z} / n \mathbf{Z}$ is isomorphic to $\mathbf{Z} / 2^{\mathrm{val}_{2}(n)} \mathbf{Z} \times \mathbf{Z} /\left(n / 2^{\mathrm{val}_{2}(n)}\right) \mathbf{Z}$ by the Chinese theorem, it follows that $\left(G, b_{q}\right)$ and $\left(K_{f}, L_{f}\right)$ both have an orthogonal summand isomorphic to $\mathbf{Z} / 2^{\mathrm{val}_{2}(n)} \mathbf{Z}$.

Remark. In the general case, i.e. $(G, q)$ possibly degenerate, $\gamma(G \otimes V, q \otimes f)=0$ if and only if $(\tilde{G}, \tilde{q})$ and $\left(K_{f}, L_{f}\right)$ have an isomorphic 2-cyclic orthogonal summand, with the same notation as in lemma 2.5 . 
Proof of Theorem 6. Proof of $(1) \Longleftrightarrow(2)$. By our hypothesis on $q, \gamma(G, q) \neq 0$. Hence $\tau(M ; G, q)$ is 0 if and only if $\gamma\left(G \otimes H_{2}(X ; \mathbf{Z})\right)$ is 0 . Now apply lemma 3.4.

Proof of $(2) \Longleftrightarrow(3)$. Assume first that both $\left(T, \mathcal{L}_{M}\right)$ and $\left(G, b_{q}\right)$ have an orthogonal summand of order $2^{k}$. Denote that of $T$ by $\langle a\rangle$ for some element $a \in T$. Since $\langle a\rangle$ is an orthogonal summand of $T$ and $\mathcal{L}_{M}$ is non-degenerate on $T$, the restriction $\mathcal{L}_{M} \mid\langle a\rangle \times\langle a\rangle$ is non-degenerate. Thus $a$ determines an element $\alpha \in \operatorname{Hom}\left(T, \mathbf{Z} / 2^{k} \mathbf{Z}\right) \subset$ $\operatorname{Hom}\left(H_{1}(M ; \mathbf{Z}), \mathbf{Z} / 2^{k} \mathbf{Z}\right)$ by $\mathcal{L}_{M}(a, x)=\frac{\alpha(x)}{2^{k}}$. Since $\operatorname{Hom}\left(H_{1}(M ; \mathbf{Z}), \mathbf{Z} / 2^{k} \mathbf{Z}\right) \cong$ $H^{1}\left(M ; \mathbf{Z} / 2^{k} \mathbf{Z}\right)$, we view $\alpha$ as an element in $H^{1}\left(M ; \mathbf{Z} / 2^{k} \mathbf{Z}\right)$ and apply Turaev's formula [Tu2, Theorem I]:

$$
\frac{1}{2^{k}}(\alpha \cup \alpha \cup \alpha)[M](\bmod 1)=2^{k-1} \mathcal{L}_{M}(a, a) \neq 0 .
$$

Conversely, if $K \cong \mathbf{Z} / 2^{k} \mathbf{Z}$ is an orthogonal summand of $G$ such that the inequality above holds, there exists an element $a \in T$ of order $2^{k}$ such that $\left.\mathcal{L}_{M}\right|_{\langle a\rangle \times\langle a\rangle}$ (where $\langle a\rangle$ denotes the subgroup generated by $a$ in $T$ ) is non-degenerate. Then it follows from [Wa, lemma (1)] that $\langle a\rangle$ is an orthogonal summand of $T$. It is isomorphic to $K$.

\section{Proof of the Reciprocity formula}

Outline of the proof. We interpret the reciprocity formula as an identity involving a bilinear pairing (see lemma 4.2). Using a stabilization argument (lemma 4.3), we reduce the reciprocity formula to an identity between classical Gauss sums, already known to Cauchy and Kronecker (relation (8)).

We shall prove the following version of the reciprocity formula.

Theorem 7. Denote by $(*)$ the following condition: $\left(K_{f}, L_{f}\right)$ and $\left(K_{g}, L_{g}\right)$ have isomorphic 2-cyclic orthogonal summands. Then

$$
\begin{aligned}
\gamma\left(K_{f}\right. & \left.\otimes W, \phi_{f, v} \otimes g\right) \cdot \gamma\left(K_{g} \otimes V, \phi_{g, w} \otimes f\right) \\
& = \begin{cases}0 & \text { if }(*), \\
e^{\frac{\pi i}{4}(\sigma(f) \sigma(g)-f(v, v) g(w, w))} & \text { otherwise. }\end{cases}
\end{aligned}
$$

In the case when $(*)$ is satisfied, it follows from lemma 3.4 that

$$
\gamma\left(K_{f} \otimes W, \phi_{f, v} \otimes g\right)=\gamma\left(K_{g} \otimes V, \phi_{g, w} \otimes f\right)=0
$$

and therefore $(13)$ holds. In the case when $(*)$ is not satisfied, again by lemma 3.4 , the formula (23) is equivalent to (13).

Since the reciprocity formula is verified in the case $(*)$, we assume from now on that $\left(K_{f}, L_{f}\right)$ and $\left(K_{g}, L_{g}\right)$ have no isomorphic 2-cyclic orthogonal summands.

Lemma 4.1. Let $q: G \rightarrow \mathbf{Q} / \mathbf{Z}$ be a hyperbolic quadratic form. There exists $(f, v) \in \mathfrak{M}_{\mathbf{Z}}^{\mathrm{Wu}}$ such that $\phi_{f, v}=q, \sigma(f)=0$ and $f(v, v) \equiv 0 \bmod 8$.

Proof. The fact that there exists $(f, v) \in \mathfrak{M}_{\mathbf{Z}}^{\mathrm{Wu}}$ such that $\phi_{f, v}=q$ follows from the surjectivity of the map $\phi$ (see lemma 2.1, (c)). Since $L_{f}$ is the bilinear form associated to $\phi_{f, v}, L_{f}$ is hyperbolic. Among the integral forms $g$ such that $L_{g}=L_{f}$, choose one of minimal rank, as in the proof of lemma 2.8. The signature of such a form is 0 . Use lemma 2.1, (b), to equip it with a Wu class such that its image by $\phi$ is still $q$. Next, it follows from Van der Blij's formula [Bl] (as formulated in [BM]) that

$$
\gamma\left(K_{f}, \phi_{f, v}\right)=e^{\frac{\pi i}{4}(\sigma(f)-f(v, v))}=e^{-\frac{\pi i}{4} f(v, v)} .
$$


On the other hand, from lemma $2.7 \gamma\left(K_{f}, \phi_{f, v}\right)=1$. The comparison of these two equalities leads to $f(v, v) \equiv 0 \bmod 8$.

Given two integral forms $f: V \times V \rightarrow \mathbf{Z}$ and $g: W \times W \rightarrow \mathbf{Z}$ equipped with Wu classes $v$ and $w$ respectively, denote by $\mathcal{F}((f, v),(g, w))$ (or simply by $\mathcal{F}(f, g)$, if no confusion is likely to occur) the product

$$
e^{-\frac{\pi i}{4}(\sigma(f) \sigma(g)-f(v, v) g(w, w))} \gamma\left(K_{f} \otimes W, \phi_{f, v} \otimes g\right) \gamma\left(K_{g} \otimes V, \phi_{g, w} \otimes f\right) .
$$

The next lemma sets up the framework in which formula (23) is interpreted.

Lemma 4.2. The map $\mathcal{F}: \mathfrak{M}_{\mathbf{Z}}^{\mathrm{Wu}} \times \mathfrak{M}_{\mathbf{Z}}^{\mathrm{Wu}} \rightarrow \mu_{8} \cup\{0\}$,

$$
((f, v),(g, w)) \mapsto \mathcal{F}((f, v),(g, w))
$$

induces a bilinear pairing $\widehat{\mathfrak{M Q}} \times \widehat{\mathfrak{M Q}} \rightarrow \mu_{8} \cup\{0\}$ such that the following diagram is commutative:

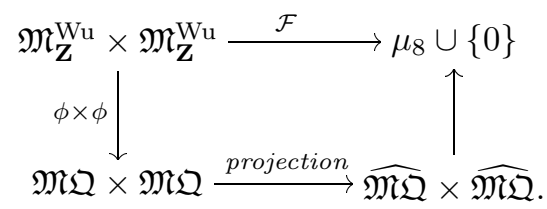

Proof. The first step consists in establishing that $\mathcal{F}((f, v),(g, w))$ only depends on $\phi_{f, v}$ and $\phi_{g, w}$. Let us show that if $(g, w)$ is fixed in $\mathfrak{M}_{\mathbf{Z}}^{\text {Wu }}$, then $\mathcal{F}((f, v),(g, w))$ only depends on $\phi_{f, v}$. Using lemma 2.1, (b), it is sufficient to show that $\mathcal{F}((f, v),(g, w))$ $=\mathcal{F}\left(\left(f^{\prime}, v^{\prime}\right),(g, w)\right)$ where $\left(f^{\prime}, v^{\prime}\right)=\left(f \oplus( \pm 1), v \oplus v_{0}\right)$ where $v_{0}$ is an odd integer. We obtain: $\phi_{f^{\prime}, v^{\prime}}=\phi_{f, v}$ and

$$
\sigma\left(f^{\prime}\right) \sigma(g)-f^{\prime}\left(v^{\prime}, v^{\prime}\right) g(w, w)=\sigma(f) \sigma(g)-f(v, v) g(w, w) \pm(\sigma(g)-g(w, w)) .
$$

Thus, using the multiplicativity of $\gamma$ and the equality

$$
e^{ \pm \frac{\pi i}{4}(\sigma(g)-g(w, w))}=\gamma\left(K_{g}, \pm \phi_{g, w}\right),
$$

we deduce that $\mathcal{F}\left(\left(f^{\prime}, v^{\prime}\right),(g, w)\right)=\mathcal{F}((f, v),(g, w))$. Since $(f, v)$ and $(g, w)$ play symmetric roles, this proves the first step. Furthermore, since $\phi: \mathfrak{M}_{\mathbf{Z}}^{W u} \rightarrow \mathfrak{M Q}$ is a homomorphism, the map $\widehat{\mathfrak{M Q}} \times \widehat{\mathfrak{M Q}} \rightarrow \mu_{8} \cup\{0\}$ through which $\mathcal{F}$ factors is a bilinear pairing. To conclude, since $(f, v)$ and $(g, w)$ play symmetric roles, it suffices to show that $\mathcal{F}((f, g),(g, w))=1$ if $\phi_{f, v}$ is hyperbolic. By lemma 2.7, $\gamma\left(K_{f} \otimes W, \phi_{f, v} \otimes g\right)=1$. We apply lemma 4.1 so that we can assume that $\sigma(f)=0$ and $f(v, v) \equiv 0 \bmod 8$. Thus

$$
e^{-\frac{\pi i}{4}(\sigma(f) \sigma(g)-f(v, v) g(w, w))} \gamma\left(K_{g} \otimes V, \phi_{g, w} \otimes f\right)=\gamma\left(K_{g} \otimes V, \phi_{g, w} \otimes f\right)=1,
$$

where the last equality follows from lemma 2.8. This achieves the proof.

Lemma 4.2 says that the equality we want to prove should be understood as a relation between invariants of $\widehat{\mathfrak{M Q}}$. To achieve the proof, it suffices to prove that $\mathcal{F}(f, g)=1$.

As another step towards (23), we observe that the special case of (23) when $f$ and $g$ are 1-dimensional (i.e., the lattices $V$ and $W$ both have rank equal to 1 ) is exactly given by formula (8).

The following result is a tool to reduce (23) to the 1-dimensional case already treated in (8); it is a variation of a lemma due to T. Ohtsuki [Oh]. Recall that the 
2-valuation of an integer $m$, denoted $v_{2}(m)$, is the greatest nonnegative integer $n$ such that $2^{n}$ divides $m$.

Lemma 4.3 (Stabilization). Let $(G, L) \in \widehat{\mathfrak{M}}$. There exist positive integers $a_{1}, \ldots$, $a_{r}$ and $b_{1}, \ldots, b_{s}$ such that the following identity holds in $\widehat{\mathfrak{M}}$ :

$$
(G, L) \oplus\left(\frac{ \pm 1}{a_{1}}\right) \oplus \cdots \oplus\left(\frac{ \pm 1}{a_{r}}\right)=\left(\frac{ \pm 1}{b_{1}}\right) \oplus \cdots \oplus\left(\frac{ \pm 1}{b_{s}}\right) .
$$

Furthermore, one can impose the following condition: if a representative pairing $(G, L)$ has no orthogonal cyclic summand of order $2^{k}$, then one can choose the integers $a_{1}, \ldots, a_{r}$ and $b_{1}, \ldots, b_{s}$ in such a way that their 2-valuation is different from $k$.

See $\S 2.1$ for notation. Roughly speaking, lemma 4.3 says that by adding cyclic pairings, one can diagonalize $(G, L)$ in $\widehat{\mathfrak{M}}$. We say that the relation $(24)$ is a stabilization of $(G, L)$. This stabilization argument really relies on the algebraic structure of $\widehat{\mathfrak{M}}$.

Proof. According to [Wa], the form $L$ is a direct sum of $\left(\frac{a}{b}\right)$ with $a$ and $b$ coprime such that $0<|a|<b$ and $E_{0}^{l}$ for $1 \leq l$ and $E_{1}^{l}$ for $2 \leq l$ (see the end of $\S 2.1$ for notation). Since $E_{0}^{l}$ is hyperbolic, it is 0 in $\widehat{\mathfrak{M}}$ and it suffices to treat the two other cases. Consider the case $L=\left(\frac{a}{b}\right)$ first. Using the identity [Mu, proof of lemma 2.2]

$$
\left(\frac{a}{b}\right) \oplus\left(\frac{\operatorname{sign}(a)}{|a| b}\right)=\left(\frac{1}{|a|}\right) \oplus\left(\frac{1}{|a|}\right) \oplus\left(\frac{\operatorname{sign}(a) b}{|a|}\right)
$$

we deduce by induction that there exist integers $a_{1}, \ldots, a_{r}$ and $b_{1}, \ldots, b_{s}$, such that in $\mathfrak{M}$,

$$
\left(\frac{a}{b}\right) \oplus\left(\frac{ \pm 1}{a_{1}}\right) \oplus \cdots \oplus\left(\frac{ \pm 1}{a_{r}}\right)=\left(\frac{ \pm 1}{b_{1}}\right) \oplus \cdots \oplus\left(\frac{ \pm 1}{b_{s}}\right) .
$$

If $(G, L)$ has no orthogonal cyclic summand of order $2^{k}$, then $v_{2}(b) \neq k$. It is then clear from (25) and the identity

$$
\left(\frac{a}{b}\right)=\left(\frac{a-b}{b}\right)
$$

that we can require $a_{1}, \ldots, a_{r}$ and $b_{1}, \ldots, b_{s}$ to be of valuation different from $k$. Consider next the case $L=E_{1}^{l}$ for some $2 \leq l$. If $l \neq k$, we use the following relations (cf. relations $(0.3)$ in $[\mathrm{KK}])$ in $\mathfrak{M}$ :

$$
\begin{aligned}
& E_{1}^{l} \oplus\left(\frac{3}{2^{l}}\right)=\left(\frac{1}{2^{l}}\right) \oplus\left(\frac{1}{2^{l}}\right) \oplus\left(\frac{1}{2^{l}}\right) \quad \text { for } l \geq 3, \\
& E_{1}^{2} \oplus\left(\frac{-1}{4}\right)=\left(\frac{1}{4}\right) \oplus\left(\frac{1}{4}\right) \oplus\left(\frac{1}{4}\right) \quad \text { for } l=2 .
\end{aligned}
$$

If $l=k$, then we use the relation (cf. relation (1.3) in [KK]):

$$
E_{1}^{k} \oplus\left(\frac{1}{2^{k+1}}\right)=E_{0}^{k} \oplus\left(\frac{-3}{2^{k+1}}\right)
$$

And one can use once more relation (26) to stabilize $\left(\frac{-3}{2^{k+1}}\right)$, that is,

$$
\left(\frac{-3}{2^{k+1}}\right) \oplus\left(\frac{-1}{3 \cdot 2^{k+1}}\right)=\left(\frac{1}{3}\right) \oplus\left(\frac{1}{3}\right) \oplus\left(\frac{(-1)^{k}}{3}\right)
$$


(the $(-1)^{k}$ on the right hand side is the residue modulo 3 of $-2^{k+1}$ ), which combined with (29) yields the desired equality. This finishes the proof.

Lemma 4.4. Assume that $f$ or $g$ is 1-dimensional, that is $V$ or $W$ has rank 1. Then $\mathcal{F}(f, g)=1$, i.e., formula (23) holds.

Proof. Suppose, with no loss of generality, that $g$ is 1-dimensional. Then $K_{g}$ is a cyclic group. Let $k$ be the 2 -valuation of its order. Since $\left(K_{f}, L_{f}\right)$ has no orthogonal cyclic summand of order $2^{k}$, we apply lemma 4.3 to stabilize $\left(K_{f}, L_{f}\right)$. There exist positive integers $a_{1}, \ldots, a_{r}$ and $b_{1}, \ldots, b_{s}$ such that

$$
\left(K_{f}, L_{f}\right) \oplus\left(\frac{ \pm 1}{a_{1}}\right) \oplus \cdots \oplus\left(\frac{ \pm 1}{a_{r}}\right)=\left(\frac{ \pm 1}{b_{1}}\right) \oplus \cdots \oplus\left(\frac{ \pm 1}{b_{s}}\right),
$$

where all numbers $a_{1}, \ldots, a_{r}$ and $b_{1}, \ldots, b_{s}$ are of valuation different from $k$. Choose $\mathrm{Wu}$ classes $u_{1}, \ldots, u_{r} \in \mathbf{Z}$ for the forms $\left( \pm a_{1}\right), \ldots,\left( \pm a_{r}\right)$ respectively, and $\mathrm{Wu}$ classes $u_{1}^{\prime}, \ldots, u_{s}^{\prime} \in \mathbf{Z}$ for the forms $\left( \pm b_{1}\right), \ldots,\left( \pm b_{s}\right)$ respectively. Then $z=v \oplus \bigoplus_{j=1}^{r} u_{j}$ (recall $v$ is a Wu class for $f$ ) is a Wu class for $f \oplus \bigoplus_{j=1}^{r}\left( \pm a_{j}\right)$ and $z^{\prime}=\bigoplus_{j=1}^{s} u_{j}^{\prime}$ is a $\mathrm{Wu}$ class for $\bigoplus_{j=1}^{s}\left( \pm b_{j}\right)$. For this choice of $\mathrm{Wu}$ classes and by additivity of $\mathrm{Wu}$ classes with respect to direct sums, we apply $\mathcal{F}(\cdot, g)$ to $(30)$ and obtain:

$$
\mathcal{F}(f, g) \mathcal{F}\left(\left( \pm a_{1}\right), g\right) \cdots \mathcal{F}\left(\left( \pm a_{r}\right), g\right)=\mathcal{F}\left(\left( \pm b_{1}\right), g\right) \cdots \mathcal{F}\left(\left( \pm b_{s}\right), g\right) .
$$

Since $\left( \pm a_{j}\right)$ (resp. $\left( \pm b_{j}\right)$ and $g$ are both 1-dimensional forms, $\mathcal{F}\left(\left( \pm a_{j}\right), g\right)=1$ (resp. $\left.\mathcal{F}\left(\left( \pm b_{j}\right), g\right)=1\right)$. It follows that $\mathcal{F}(f, g)=1$. This is the desired result.

End of the proof. Since $\mathcal{F}$ is bilinear (bimultiplicative with respect to orthogonal sums), we can assume that $\left(K_{f}, L_{f}\right)$ and $\left(K_{g}, L_{g}\right)$ are irreducible. Hence $K_{f}$ (resp. $K_{g}$ ) is either a $p$-group, where $p \geq 2$ is prime, or a product of two copies of a 2cyclic group [Wa]. Since by hypothesis $\left(K_{f}, L_{f}\right)$ and $\left(K_{g}, L_{g}\right)$ have no isomorphic orthogonal 2-cyclic summands, we deduce that one of them, for example $\left(K_{g}, L_{g}\right)$, has no orthogonal cyclic summand of order $2^{k}$, where $k$ is the 2 -valuation of the exponent of $K_{f}$. Then we apply lemma 4.3 to $\left(K_{g}, L_{g}\right)$. There exist positive integers $a_{1}, \ldots, a_{r}$ and $b_{1}, \ldots, b_{s}$ such that

$$
\left(K_{g}, L_{g}\right) \oplus\left(\frac{ \pm 1}{a_{1}}\right) \oplus \cdots \oplus\left(\frac{ \pm 1}{a_{r}}\right)=\left(\frac{ \pm 1}{b_{1}}\right) \oplus \cdots \oplus\left(\frac{ \pm 1}{b_{s}}\right),
$$

where the 2-valuations of $a_{1}, \ldots, a_{r}, b_{1}, \ldots, b_{s}$, respectively, are different from $k$. Choose $\mathrm{Wu}$ classes for the forms $\left( \pm a_{j}\right), 1 \leq j \leq r$ and $\left( \pm b_{j}\right), 1 \leq j \leq s$ respectively. For this choice of $\mathrm{Wu}$ classes and by additivity of $\mathrm{Wu}$ classes with respect to direct sums, we obtain

$$
\mathcal{F}(f, g) \mathcal{F}\left(f,\left( \pm a_{1}\right)\right) \cdots \mathcal{F}\left(f,\left( \pm a_{r}\right)\right)=\mathcal{F}\left(f,\left( \pm b_{1}\right)\right) \cdots \mathcal{F}\left(f,\left( \pm b_{s}\right)\right) .
$$

Lemma 4.4 yields $\mathcal{F}\left(f,\left( \pm a_{j}\right)\right)=1$ for $1 \leq j \leq r$ and $\mathcal{F}\left(f,\left( \pm b_{j}\right)\right)=1$ for $1 \leq j \leq s$. It follows that $\mathcal{F}(f, g)=1$. This finishes the proof of Theorem 7 .

Remark. The following formula, due to Cauchy and Kronecker, is more general than (8):

$$
|b|^{-\frac{1}{2}} \sum_{x \in \mathbf{Z} / b \mathbf{Z}} e^{\pi i \frac{a}{b}(x+u)^{2}}=e^{\frac{\pi i}{4} \operatorname{sign}(a b)}|a|^{-\frac{1}{2}} \sum_{x \in \mathbf{Z} / a \mathbf{Z}} e^{-\pi i \frac{b}{a} x^{2}+2 \pi i u x}
$$

where $a$ and $b$ are nonzero integers, $u \in \mathbf{Q}$, such that $a b+2 a u \in 2 \mathbf{Z}$. We note that (8) can be deduced from the formula above by substituting $u=\frac{b}{2}$ but (31) is not 
a particular case of (13). We conjecture that there exists a formula, analogous to (13), which does generalize both (9) and (31).

\section{Appendix A. Relation With modular categories}

We explain how the invariants $\tau(M ; G, q)$ arise from the theory of modular categories. We first give a brief survey of this theory (we refer to [Tu1] for more details) and then we describe the relation with our work.

Modular categories are tensor categories with certain additional algebraic structures (braiding and twist) and properties of semisimplicity and finiteness. Semisimplicity and finiteness mimic the corresponding properties in the representation theory of semisimple Lie algebras. In particular, simple objects play the role of irreducible modules. The braiding is a generalization of the permutation isomorphism $U \otimes V \rightarrow V \otimes U$ for modules over a commutative ring. Given a tensor (monoidal) category $\mathcal{V}$, a braiding is a family of isomorphisms

$$
c=\left\{c_{U, V}: U \otimes V \rightarrow V \otimes U\right\}_{U, V \in \mathcal{V}}
$$

which satisfy some naturality and compatibility conditions. A twist in $\mathcal{V}$ is a family of isomorphisms

$$
\theta=\left\{\theta_{U}: U \rightarrow U\right\}_{U \in \mathcal{V}}
$$

which satisfy the identity

$$
\theta_{U \otimes V}=c_{V, U} c_{U, V}\left(\theta_{U} \otimes \theta_{V}\right)
$$

for any objects $U$ and $V$ in $\mathcal{V}$. As all the algebraic formalism involved in the theory, the braiding and twist are best seen graphically, once a proper connection between ribbon graphs (or colored framed links) and ribbon categories is established. A ribbon category is a monoidal category with braiding and twist plus one more feature which generalizes the usual duality in linear algebra. From a ribbon category $\mathcal{V}$, one can construct a certain category of ribbon graphs $R i b_{\mathcal{V}}$, which consists of geometric objects. There is a canonical functor $R i b \mathcal{V} \rightarrow \mathcal{V}$ which "represents" geometric framed links (more generally ribbon graphs) in terms of the ribbon category $\mathcal{V}$ we started with. Furthermore, this functor yields isotopy invariants of framed links in $\mathbf{R}^{3}$. Using properties of semisimplicity and finiteness, one derives from this functor an invariant of closed oriented 3-manifolds.

Let $G$ be a multiplicative finite abelian group equipped with a bilinear form $c: G \times G \rightarrow U(1)$ (here $U(1)$ denotes the unit group of complex numbers of absolute value 1 ). The form $c$ induces a quadratic form $q_{c}: G \rightarrow \mathbf{Q} / \mathbf{Z}$ by $q_{c}(x)=$ $\exp (2 \pi i c(x, x))$ for any $x \in G$. Using this form and presenting $M$ as the result of surgery in $S^{3}$, we can define an invariant $\tau\left(M ; G, q_{c}\right)$ by $(3)$. This invariant $M \mapsto$ $\tau\left(M ; G, q_{c}\right)$ coincides with the one coming from the following modular category $\mathcal{V}$ (see [Tu1, p. 29]): objects are elements of $G$ (written multiplicatively); for $g, h \in G$, the set of morphisms $g \rightarrow h$ is a copy of $\mathbf{C}$ if $g=h$ and is $\{0\}$ otherwise; the composition of morphisms is defined as the product of the corresponding elements in $\mathbf{C}$; the tensor product of objects is their product in $G$. This category is a strict monoidal category. For $g, h \in G$, the braiding $g h \rightarrow h g$ is defined to be the element $c(g, h) \in \mathbf{C}$; the twist $g \rightarrow g$ is defined to be $c(g, g) \in \mathbf{C}$. If, moreover, we define the duality by $g^{\star}=g^{-1}$ for all $g \in G$, then this category becomes an abelian ribbon category. It can be seen that the category is modular if and only if the $S$-matrix $(c(g, h) c(h, g))_{g, h \in G}$ is invertible over C. Under this condition, the invariant $\tau_{\mathcal{V}}$ 
coming from the category $\mathcal{V}$ is essentially the same as our invariant $\tau\left(M ; G, q_{c}\right)$. More precisely, the following relation holds:

$$
\tau_{\mathcal{V}}\left(M ; G, q_{c}\right)=|G|^{-\frac{1}{2}} \cdot \tau\left(M ; G, q_{c}\right) .
$$

In other words, the invariant $M \mapsto \tau\left(M ; G, q_{c}\right)$ comes from the modular category $\mathcal{V}$ if $q_{c}$ is non-degenerate. On the other hand, a weaker condition than the invertibility of the $S$-matrix is known ([Tu3]): one can associate an invariant of closed oriented three-manifolds to a semisimple category if $\Delta_{\mathcal{V}} \Delta_{\overline{\mathcal{V}}} \neq 0$ where $\Delta_{\mathcal{V}}$ is a certain element of the ground ring of the category $\mathcal{V}$ and where $\overline{\mathcal{V}}$ denotes the mirror category of $\mathcal{V}$. In our case, $\Delta_{\mathcal{V}}=\sum_{x \in G} e^{-2 \pi i q_{c}(x)}$ and $\Delta_{\overline{\mathcal{V}}}=\sum_{x \in G} e^{2 \pi i q_{c}(x)}$ (because the category is hermitian) so the above condition amounts to the nonnullity of $\gamma(G, q)$ and we recover all invariants $M \mapsto \tau\left(M ; G, q_{c}\right)$ in this way. We see in particular that different braidings $c$ and $c^{\prime}$ may give rise to the same invariant; this happens if and only if $c(x, x)=c^{\prime}(x, x)$ for all $x \in G$.

\section{Appendix B. Generalization to Closed oriented ( $4 n-1)$-manifolds}

At first sight, or as the construction from the theory of modular categories maybe would suggest, the definition of the invariant $\tau(M ; G, q)$ seems to be rather specific to dimension 3. However, Theorem 4 enables us to define such an invariant for $(4 n-1)$-manifolds as well. More precisely, let $M$ be a closed oriented $(4 n-1)$ manifold. There is a well defined linking form of $M, \mathcal{L}_{M}: T \times T \rightarrow \mathbf{Q} / \mathbf{Z}$, where $T=$ Tors $H_{2 n-1}(M ; \mathbf{Z})$, which is a non-degenerate, symmetric, bilinear pairing. We define $\tau(M ; G, q)$ by (16). In the case when $M=\partial W$ where $W$ is a compact, oriented $4 n$-manifold, we obtain a reciprocity formula between the intersection form $B_{W}$ on $H_{2 n}(W ; \mathbf{Z})$ (or on the free part of $H_{2 n}(W ; \mathbf{Z})$ ) and the linking form $T$. Let $f: F \otimes F \rightarrow \mathbf{Z}$ be a symmetric bilinear form on a finitely generated free abelian group $F$, with a Wu class $w \in F$, such that $\left(K_{f}, \phi_{f, v}\right)=(G, q)$ and let $Q: T \rightarrow \mathbf{Q} / \mathbf{Z}$ be a quadratic form over $\mathcal{L}_{M}$. Then

$$
\overline{\gamma(G, q)}^{\sigma\left(B_{W}\right)} \gamma\left(G \otimes H_{2 n}(W ; \mathbf{Z}), q \otimes B_{W}\right)=\overline{\gamma(T, Q)}^{f(v, v)} \gamma(T \otimes F, Q \otimes f) .
$$

\section{REFERENCES}

[Be] R. Bellman, A brief introduction to theta functions, Holt, Rinehart \& Winston, New York, 1961. MR 23:A2556

[BE] B. C. Berndt and R. J. Evans, The determination of Gauss sums, Bull. of the Amer. Math. Soc. 5, n. 2 (1981), 107-129. MR 82h:10051

[Bl] F. van der Blij, An invariant of quadratic forms mod 8, Indag. Math. 21 (1959), 291-293. MR 21:7183

[Br] H. Braun, Geschlechter quadratischer Formen, Journal für Math., Bd 182, H. 1. (1940), 32-49. MR 2:36f

[BM] G. Brumfield and J. Morgan, Quadratic functions, the index modulo 8 and $a \mathbf{Z} / 4 \mathbf{Z}$ Hirzebruch formula, Topology 12 (1973), 105-122. MR 48:3059

[Ch] K. Chandrasekharan, Elliptic functions, Grundlehren der mathematischen Wissenschften 281, Berlin, Heidelberg, New York, Springer Verlag, 1985. MR 87e:11058

[Dab] R. Dabrowski, Multivariate Gauss sums, preprint, Columbia University 1995.

[Del] F. Deloup, Linking forms, reciprocity for Gauss sums and invariants of 3-manifolds, prépublication de l'IRMA no. 26, Strasbourg 1996. CMP 98:08

[Du] A. H. Durfee, Bilinear and quadratic forms on torsion modules, Adv. in Math. 25 (1977), 133-164. MR 58:506

[Fr] A. Fröhlich, Hermitian and quadratic forms over rings with involution, Quart. J. Math. Oxford 20 (1969), 297-317. MR 40:5642 
[KK] A. Kawauchi and S. Kojima, Algebraic classification of linking pairings on 3-manifolds, Math. Ann. 253 (1980), 29-42. MR 82b:57007

[Ki1] R. Kirby, A calculus for framed links in $S^{3}$, Invent. Math. 45 (1978), 35-56. MR 57:7605

[Ki2] R. Kirby, The topology of 4-manifolds, Lectures Notes in Mathematics 1374, Berlin, New York, Springer Verlag, 1989. MR 90j:57012

[Kr] A. Krazer, Zur Theorie der mehrfachen Gaußschen Summen, H. Weber Festschrift, Leipzig (1912), s. 181.

[Ky] R. Kyle, Branched covering spaces and the quadratic forms of links, Ann. of Math. 59 (1954), 539-548. MR 15:979a

[La] J. Lannes, Formes quadratiques d'enlacement sur l'anneau des entiers d'un corps de nombres, Ann. Sci. Ecole Norm. Sup., 4ème série 8 (1975), 535-579. MR 54:231

$[\mathrm{MH}]$ J. Milnor and D. Husemoller, Symmetric bilinear forms, Ergebnisse der Math. 73, Berlin, Heidelberg, New York, 1973. MR 58:22129

[MPR] J. Mattes, M. Polyak, N. Reshetikhin, On invariants of 3-manifolds derived from abelian groups, in Quantum Topology, ed. L. Kauffman, R. Baadhio, World Scientific, 1993. MR 95e:57031

[MOO] H. Murakami, T. Ohtsuki, M. Okada, Invariants of three-manifolds derived from linking matrices of framed links, Osaka J. Math. 29 (1992), 545-572. MR 93h:57013

$[\mathrm{Mu}] \mathrm{H}$. Murakami, Quantum SO(3)-invariants dominate the SU(2)-invariant of Casson and Walker, preprint, Osaka University, 1992.

[Oh] T. Ohtsuki, A polynomial invariant of rational homology 3-spheres, preprint, 1994.

[Rok] V. Rokhlin in A la recherche de la topologie perdue, Progress in Mathematics, vol. 62, ed. L. Guillou, A. Marin, Birkhäuser, 1986. MR 88k:57002

[Rol] D. Rolfsen, Knots and Links, Publish or Perish, Inc, Berkeley, 1976. MR 58:24236

[Sa] C.-H. Sah, Symmetric bilinear forms and quadratic forms, J. Algebra 20 (1972), 144-160. MR 45:3448

[Sc] W. Scharlau, Quadratic and hermitian forms, Heidelberg, New York, Tokyo, SpringerVerlag, 1986.

[Si] C. L. Siegel, Uber die analytische Theorie der quadratischen Formen, Ann. Math., 36 (1935), 527.

[Sp] T. A. Springer, Caractères quadratiques de groupes abéliens finis et sommes de Gauss, Colloque sur les formes quadratiques (1975, Montpellier), Bull. Soc. Math. France, mémoire 48 (1976), 103-115. MR 58:27762

[Tu1] V. Turaev, Quantum invariants of knots and 3-manifolds, De Gruyter, Studies in Math., 1994. MR 95k:57014

[Tu2] V. Turaev, Cohomology rings, linking forms and invariants of spin structures of threedimensional manifolds, Math. USSR Sbornik, Vol. 48 (1984) No.1.

[Tu3] V. Turaev, private conversation, 1995.

[Wa] C.T.C Wall, Quadratic forms on finite groups and related topics, Topology 2 (1963), 281-298. MR 28:133

Institut de Recherche en Mathématiques Avancées, 7, Rue René Descartes, 67084 Strasbourg, France

Current address: Laboratoire de Mathématiques, Emile Picard, Université Paul Sabatier, Toulouse III, 118, route de Narbonne, 31062 Toulouse, France

E-mail address: deloup@math.u-strasbg.fr 